\title{
A global collisionless PIC code in magnetic coordinates
}

\author{
S. Jolliet ${ }^{\mathrm{a}, *}$, A. Bottino ${ }^{\mathrm{b}}$, P. Angelino ${ }^{\mathrm{a}}, \mathrm{R}^{\mathrm{R}}$ Hatzky $^{\mathrm{c}}$, \\ T. M. Tran ${ }^{\text {a }}$, B. F. Mcmillan ${ }^{\text {a }}$, O. Sauter ${ }^{\text {a }}$, Y. Idomura ${ }^{\mathrm{d}}$, \\ L. Villard ${ }^{\mathrm{a}}$ \\ ${ }^{a}$ Ecole Polytechnique Fédérale de Lausanne \\ Centre de Recherches en Physique des Plasmas \\ Association Euratom-Confédération Suisse \\ CH-1015 Lausanne, Switzerland \\ ${ }^{\mathrm{b}}$ Max Planck Institut für Plasmaphysik \\ IPP-EURATOM Association, Garching, Germany \\ ${ }^{\mathrm{c}}$ Rechenzentrum der Max-Planck-Gesellschaft und des Max-Planck-Institutes für \\ Plasmaphysik, EURATOM-Association, D-85748, Germany \\ dJapan Atomic Energy Agency, Higashi-Ueno 6-9-3, Taitou, Tokyo 110-0015, \\ Japan
}

\begin{abstract}
A global plasma turbulence simulation code, ORB5, is presented. It solves the gyrokinetic electrostatic equations including zonal flows in axisymmetric magnetic geometry. The present version of the code assumes a Boltzmann electron response on magnetic surfaces. It uses a Particle-In-Cell (PIC), $\delta f$ scheme, $3 \mathrm{D}$ cubic B-splines finite elements for the field solver and several numerical noise reduction techniques. A particular feature is the use of straight-field-line magnetic coordinates and a field aligned Fourier filtering technique that dramatically improves the performance of the code in terms of both the numerical noise reduction and the maximum time step allowed. Anoter feature is the capability to treat arbitrary axisymmetric ideal MHD equilibrium configurations. The code is heavily parallelized, with scalability demonstrated up to 4096 processors and $10^{9}$ marker particles. Various numerical convergence tests are performed. The code is validated against an analytical theory of zonal flow residual, geodesic acoustic oscillations and damping, and against other codes for a selection of linear and nonlinear tests.
\end{abstract}

Key words: gyrokinetics, PIC, micro-instabilities, ITG, zonal flows PACS: 52.30.Gz, 52.35.Qz, 52.35.Ra 
* Corresponding author.

Email address: sebastien.jolliet@epfl.ch (S. Jolliet). 


\section{Introduction}

Anomalous transport is currently one of the most active fields of research in magnetic confinement fusion. The cross-field transport measured in Tokamak experiments exceeds the neoclassical predictions by up to two orders of magnitude for the low confinement regime (L-mode) [1]. Even in the high confinement regime (H-mode) where transport is reduced near the plasma edge, anomalous transport still persists in the core. This phenomenon is attributed to plasma turbulence [2], which is driven by micro-instabilities [3]. The latter are mainly generated by free sources of energy in the plasma such as density and temperature gradients. It is now widely believed that self-organization of turbulence plays a crucial role. It refers to a process in which the internal organization of a system increases automatically without being guided or managed by an outside source. In magnetized plasmas, self-organization mainly appears through two different structures: zonal flows [4] and large-scale transport, such as avalanches [5] and streamers $[6,7]$.

In general, a full kinetic treatment of micro-instabilities is needed. In this framework, the gyrokinetic model [8] is usually sufficient for drift wave turbulence at low frequency. It removes the gyorangle dependance in the original equations, thus reducing the phase space from $6 \mathrm{D}$ to $5 \mathrm{D}$. Various gyrokinetic equations can be found in [9-12]. Among the different approaches used to solve the gyrokinetic equations, the Particle-In-Cell (PIC) method [8,13-18] is one of the most promising schemes. The distribution function is sampled along trajectories with numerical particles (markers). The main advantage is that memory requirements are smaller than for Eulerian codes, where the distribution function is discretized on a 5D grid. Moreover, it is conceptually simple, easily generalized to multi-dimensional simulations and more adapted for complex geometries such as stellerators $[19,20]$ than Eulerian codes. However, the PIC method unavoidably gives rise to statistical noise which can lead to an unphysical behaviour in the nonlinear phase of the simulation, but significant progresses have been made to limit this effect $[21,22]$. In addition to the solving methods, another important distinction between the different models is the simulated domain. In local simulations, turbulence is studied on a single magnetic surface. A less restrictive method is to simulate a flux-tube following a given magnetic-field line. This approach reduces the computer ressources needed, but imposes inconsistent $T$ and $\nabla T$ profiles (i.e. $T=$ const, $\nabla T=$ const) and generally employs unphysical radially periodic boundary conditions. The global approach is certainly the most realistic model, because it contains the whole radial domain and therefore the effects of profile variation.

The aim of this paper is to present the ORB5 code, originally written by Parker [18] and further developed by Tran [13]. ORB5 is a nonlinear gyrokinetic global code which solves the Vlasov-Poisson system in the electrostatic and collisionless limit, and has the unique capability of handling true MHD 
equilibria [23].

The paper is organised as follows. In section 2, the gyrokinetic equations are derived. Section 3 presents the implementation of these equations and the associated numerical algorithms. Section 4 shows the parallel performance of the code. Results related to the numerical schemes and benchmarks are shown in section 5. Finally, conclusion and future works are exposed in section 6 . 


\section{Gyrokinetic model}

\subsection{Definitions and normalization}

The code ORB5 solves the Vlasov-Poisson system in the gyrokinetic limit for an axisymetric toroidal plasma. Magnetic surfaces are labelled with the poloidal flux $\psi$, or by the radial coordinate $s=\sqrt{\psi / \psi_{\text {edge }}}=\sqrt{\tilde{\psi}}$. The geometrical radial coordinate will be noted $\rho$ and the poloidal angle $\theta$. The magnetic field is defined as $\vec{B}=F(\psi) \vec{\nabla} \varphi+\vec{\nabla} \psi \times \vec{\nabla} \varphi$, where $F(\psi)$ is the poloidal current flux function. Two different kinds of magnetic equilibria are implemented: circular concentric magnetic surfaces, referred to as ad hoc equilibrium, and true MHD equilibria. For the latter case, ORB5 is coupled with the CHEASE code [24], which solves the Grad-Shafranov equation. The poloidal coordinate used is either the geometrical angle $\theta$ or the straight-field-line coordinate $\theta_{*}$ defined by

$$
\theta_{*}=\frac{1}{q(s)} \int_{0}^{\theta} \frac{\vec{B} \cdot \vec{\nabla} \varphi}{\vec{B} \cdot \vec{\nabla} \theta^{\prime}} \mathrm{d} \theta^{\prime}
$$

where $q(s)$ is the safety factor. In this paper, $\chi$ represents a general poloidal coordinate. In ORB5, the options $\chi=\theta$ and $\chi=\theta_{*}$ have been implemented. All symbols with the subscript $i$ will be used for ion quantities, while the subscript $e$ will be used for electrons. It is assumed that the plasma contains electrons and an ion species with a mass $m_{i}$ and a charge $q_{i}=e Z_{i}$. Four normalization quantities are used: $q_{i}, m_{i}, \Omega_{i}=q_{i} B_{0} / m_{i}$ and $\rho_{s}=c_{s} / \Omega_{i}$, where $B_{0}$ is the magnetic field at axis, $T_{e}\left(s_{0}\right)$ is the electron temperature in $\mathrm{eV}$ at a given reference magnetic surface $s_{0}$, and $c_{s}=\sqrt{e T_{e}\left(s_{0}\right) / m_{i}}$ is the ion sound speed.

The gyrokinetic equations solved in ORB5 are those of reference [9] which describe the evolution of the plasma in an inhomogeneous static equilibrium magnetic field. Only the electrostatic component of the perturbation is considered, and magnetic perturbations are neglected.

\subsection{Gyrokinetic Vlasov equation}

The usual gyrokinetic ordering is assumed:

$$
\begin{aligned}
\frac{\omega}{\Omega_{i}} \sim \frac{k_{\|}}{k_{\perp}} \sim \frac{e \phi}{T_{e}} \sim \frac{\rho_{L, i}}{L_{n}} \sim \frac{\rho_{L, i}}{L_{T, i}} \sim \frac{\rho_{L, i}}{L_{T, e}} & \sim \epsilon_{g}, \\
\frac{\rho_{L, i}}{L_{B}} & \sim \epsilon_{B},
\end{aligned}
$$


where $\omega$ is the characteristic fluctuation frequency, $k_{\|}$and $k_{\perp}$ are the parallel and perpendicular components of the wave vector with respect to the magnetic field, $\phi$ is the fluctuating electrostatic potential, $\rho_{L, i}$ is the ion gyroradius, and $L_{n}, L_{T, i}, L_{T, e}, L_{B}$ are the characteristic lengths associated with the density, the ion temperature, the electron temperature and the magnetic field profiles. $\epsilon_{g}$ and $\epsilon_{B}$ are the small parameters $\epsilon_{g} \sim \rho^{*}, \epsilon_{B} \sim \epsilon_{a} \epsilon_{g}$, with $\rho^{*}=\rho_{s} / a \ll 1$ and $\epsilon_{a}$ is the inverse aspect ratio. ORB5 solves the equations of motion in a $5 \mathrm{D}$ phase space $\left(\vec{R}, v_{\|}, \mu\right)$, where $\vec{R}$ is the position of the guiding center, $\mu=v_{\perp}^{2} /(2 B)$ is the magnetic moment, with the conservation property $\mathrm{d} \mu / \mathrm{d} t=0, v_{\|}$and $v_{\perp}$ are the parallel and perpendicular components of the velocity with respect to the magnetic field. The renormalised potential $\Psi\left(\vec{R}, v_{\|}, \mu\right)$ in [9] has been approximated here by the gyroaveraged electrostatic potential

$$
\Psi\left(\vec{R}, v_{\|}, \mu, t\right) \cong\langle\phi\rangle(\vec{R}, \mu, t)=\frac{1}{2 \pi} \int_{0}^{2 \pi} \phi(\vec{x}, t) \delta\left(\vec{R}+\vec{\rho}_{L, i}-\vec{x}\right) \mathrm{d} \alpha
$$

thus neglecting a term of order $\mathcal{O}\left(\epsilon_{g}^{2}\right)$. Here $\alpha$ is the gyroangle. The gyroaveraged electric field is approximated by

$$
\langle\vec{E}\rangle(\vec{R}, \mu, t) \cong-\left\langle\vec{\nabla}_{\vec{x}} \phi(\vec{x})\right\rangle(\vec{R}, \mu, t)=-\frac{1}{2 \pi} \int_{0}^{2 \pi} \mathrm{d} \alpha \tilde{\nabla}_{\tilde{\mathrm{x}}} \phi(\tilde{\mathrm{x}}),
$$

where a term of order $\mathcal{O}\left(\epsilon_{g} \epsilon_{b}\right)$ has been neglected. The polarization density is [9]:

$$
\begin{aligned}
n_{i, \text { pol }}= & \int\left\{\frac{q_{i}}{m_{i} B}(\phi-\langle\phi\rangle) \frac{\partial f}{\partial \mu}+\frac{q_{i}}{m_{i} \Omega_{i}^{2}} \vec{\nabla}\left[\int \mathrm{d} \alpha(\phi-\langle\phi\rangle)\right] \times \frac{\vec{B}}{B} \cdot \vec{\nabla} f\right\} \\
& \delta\left(\vec{R}+\vec{\rho}_{L, i}+\vec{x}\right) B_{\|}^{*} \mathrm{~d} \vec{R} \mathrm{~d} v_{\|} \mathrm{d} \mu \mathrm{d} \alpha,
\end{aligned}
$$

where $f=f\left(\vec{R}, v_{\|}, \mu\right)$ is the guiding center distribution function of the ion species, $B_{\|}^{*}=\vec{B}_{\|}^{*} \cdot \vec{B} / B$, and $\vec{B}_{\|}^{*}=\vec{B}+m_{i} v_{\|} / q_{i}(\vec{\nabla} \times \vec{h})$. The second term of Eq. (5) has been neglected, although it is of order $\mathcal{O}\left(\epsilon_{g}\right)$ : ORB5 solves the linearized quasi-neutrality equation, so when $f$ is replaced by the $f_{0}$ in the second term, it becomes smaller than the first one by a factor $\epsilon_{g}$ due to the density and temperature gradients appearing in $\vec{\nabla} f_{0}$. The gyrokinetic Vlasov equation is then:

$$
\frac{\partial f}{\partial t}+\frac{\partial f}{\partial \vec{R}} \cdot \frac{\mathrm{d} \vec{R}}{\mathrm{~d} t}+\frac{\partial f}{\partial v_{\|}} \frac{\mathrm{d} v_{\|}}{\mathrm{d} t}=0
$$

The equations of motion are given by 


$$
\begin{aligned}
\frac{\mathrm{d} \vec{R}}{\mathrm{~d} t}= & v_{\|} \vec{h}+\frac{1}{\Omega_{i} B_{\|}^{*}}\left(v_{\|}^{2}+\frac{v_{\perp}^{2}}{2}\right)(\vec{h} \times \vec{\nabla} B)-\frac{v_{\|}^{2}}{\Omega_{i} B_{\|}^{*}} \vec{h} \times[\vec{h} \times(\vec{\nabla} \times \vec{B})] \\
& +\frac{\langle\vec{E}\rangle \times \vec{B}}{B_{\|}^{*} B}, \\
\frac{\mathrm{d} v_{\|}}{\mathrm{d} t}= & \frac{1}{2} v_{\perp}^{2} \vec{\nabla} \cdot \vec{h}+\frac{v_{\perp}^{2} v_{\|}}{2 \Omega_{i} B B_{\|}^{*}}\{\vec{h} \times[\vec{h} \times(\vec{\nabla} \times \vec{B})]\} \cdot \vec{\nabla} B \\
& +\langle\vec{E}\rangle \cdot\left\{\frac{q_{i}}{m_{i}} \vec{h}+\frac{v_{\|}}{B B_{\|}^{*}}(\vec{h} \times \vec{\nabla} B)+\frac{v_{\|}}{B B_{\|}^{*}}\left(\vec{h} \times \frac{\mu_{0} \vec{\nabla} p}{B}\right)\right\},
\end{aligned}
$$

where $\vec{h}=\vec{B} / B, \mu_{0}$ is the permittivity of free space and $\vec{\nabla} p=p^{\prime}(\psi) \vec{\nabla} \psi$ is the pressure gradient. The second term of the right hand side of (8) is of order $\mathcal{O}\left(\epsilon_{B}^{2}\right)$, does not influence neither energy conservation (see section 2.5) nor the physical results and will be neglected in all the simulations. These equations of motion for a particle include the parallel motion, the drifts due to the curvature and the gradient of the magnetic field, the diamagnetic drift and a mirror term. For concentric circular magnetic surfaces, $\mu_{0} \vec{\nabla} p / B$ is replaced by $-\vec{h} \times(\vec{\nabla} \times \vec{B})$. Nonlinear terms are the $\vec{E} \times \vec{B}$ drift and the $v_{\|}$nonlinearity, which is mainly an acceleration term due to the parallel electric field.

\subsection{Background equilibrium and $\delta f$ method}

The $\delta f$ method [21] is used, i.e. the distribution function $f$ is split between a time independent part $f_{0}$ and a time dependent part $\delta f: f\left(\vec{R}, v_{\|}, \mu, t\right)=$ $f_{0}\left(\vec{R}, v_{\|}, \mu\right)+\delta f\left(\vec{R}, v_{\|}, \mu, t\right)$. A Maxwellian is chosen for $f_{0}$, i.e. $f_{0}\left(\vec{R}, v_{\|}, \mu\right)=$ $n_{0}(\Upsilon)(2 \pi)^{-3 / 2} v_{\mathrm{th}, i}^{-3}(\Upsilon) \exp \left(-\epsilon / T_{i}(\Upsilon)\right)$, where $\epsilon$ is the kinetic energy of a single ion, $\epsilon=1 / 2 m_{i} v^{2}$ and is a constant of the unperturbed motion, and $v_{\mathrm{th}, i}=$ $\sqrt{e T_{i} / m_{i}}$ is the ion thermal velocity. Three different kinds of Maxwellian can be used, depending on the choice of the variable $\Upsilon$ as either $\psi, \psi_{0}, \hat{\psi}$, where $\psi_{0}$ is the canonical toroidal momentum, $\psi_{0}=\psi+v_{\|} \Omega_{i} F(\psi) / B^{2}$ and $\hat{\psi}$ is a function of $\left(\epsilon, \mu, \psi_{0}\right)$ defined below. The axisymmetry of the tokamak implies that $\psi_{0}$ is a constant of the unperturbed motion, i.e. $\left.\frac{\mathrm{d} \psi_{0}}{\mathrm{~d} t}\right|_{0}=0$. The quantity $\hat{\psi}=\psi_{0}+\psi_{0, \text { corr }}(\epsilon, \mu)$ is another constant of motion, with

$$
\psi_{0, \mathrm{corr}}(\epsilon, \mu)=-\operatorname{sign}\left(v_{\|}\left(t_{0}\right)\right) \frac{q_{i}}{m_{i}} R_{0} \sqrt{2\left(\epsilon-\mu B_{0}\right)} \mathcal{H}\left(\epsilon-\mu B_{0}\right),
$$

where $\mathcal{H}(x)$ is the Heavyside function, therefore $\psi_{0 \text {,corr }}$ is defined only for passing particles. $\hat{\psi}$ can be seen as the closest constant of motion to $\psi$. In the 
following, $f_{0}(\psi)$ is refered as to a local Maxwellian, $f_{0}\left(\psi_{0}\right)$ as to a canonical Maxwellian and $f_{0}(\hat{\psi})$ as to a canonical Mawellian with correction. The Vlasov equation is then $\frac{\mathrm{d} \delta f\left(\vec{R}, v_{\|}, \mu\right)}{\mathrm{d} t}=-\frac{\mathrm{d} f_{0}\left(\Upsilon, v_{\|}, \mu\right)}{\mathrm{d} t}$ which can be written $\frac{\mathrm{d} \delta f\left(\vec{R}, v_{\|}, \mu\right)}{\mathrm{d} t}=$ $\tau(\vec{E})$, with

$$
\tau(\vec{E})=-f_{0}\left(\Upsilon, v_{\|}, \mu\right)\left(\left.\kappa(\Upsilon) \frac{\mathrm{d} \Upsilon}{\mathrm{d} t}\right|_{0}+\left.\kappa(\Upsilon) \frac{\mathrm{d} \Upsilon}{\mathrm{d} t}\right|_{1}-\left.\frac{q_{i}}{T(\Upsilon)}\langle\vec{E}\rangle \cdot \frac{\mathrm{d} \vec{R}}{\mathrm{~d} t}\right|_{0}\right)
$$

where $\kappa(\Upsilon)=\mathrm{d} \ln f_{0}\left(\Upsilon, v_{\|}, \mu\right) / \mathrm{d} \Upsilon$. The subscript 0 refers to the unperturbed orbits and the subscript 1 refers to the terms that depend on the perturbed electric field. In most other gyrokinetic codes, with the notable exception of the GT3D code [16], a local Maxwellian is chosen, i.e $\Upsilon=\psi$, and the first term in the r.h.s. of Eq. (10) is neglected. A local Maxwellian can lead to spurious zonal flow oscillations [16], since it is not a true equilibrium distribution function, as $\left.\frac{\mathrm{d} f_{0}^{L M}}{\mathrm{~d} t}\right|_{0} \neq 0$. When using a canonical Maxwellian, the quasi-neutrality equation is no longer satisfied as electron and ion equilibrium densities are different. In order to enforce quasi-neutrality, a radial electric field quickly develops and possibly suppresses instability. To eliminate this spurious field generation, the electron equilibrium density is further integrated from the ion distribution function after the particle loading and averaged over the poloidal angle,

$$
n_{e 0}(\psi)=\frac{1}{2 \pi} \int_{0}^{2 \pi}\left(\int f_{0}\left(\psi_{0}, \epsilon, \mu\right) \delta\left(\vec{R}+\vec{\rho}_{L, i}-\vec{x}\right) B_{\|}^{*} \mathrm{~d} \vec{R} \mathrm{~d} v_{\|} \mathrm{d} \mu \mathrm{d} \alpha\right) \mathrm{d} \theta
$$

which minimizes the difference between $n_{0 e}$ and $n_{0 i}$. Note that for small $\rho^{*}$ plasmas, there is little difference between $\psi$ and $\psi_{0}$ and the local Maxwellian becomes a better approximation. Issues related to this choice are discussed in details in Ref. [25]. When $\Upsilon=\hat{\psi}$, the approximation $\left.\left.\frac{\mathrm{d} \hat{\psi}}{\mathrm{d} t}\right|_{1} \cong \frac{\mathrm{d} \psi_{0}}{\mathrm{~d} t}\right|_{1}$ is done. As another alternative, since $f$ is constant along the trajectories, $\delta f$ can be simply obtained by $\delta f\left(\vec{R}, v_{\|}, \mu, t\right)=f\left(\vec{R}\left(t_{0}\right), v_{\|}\left(t_{0}\right), \mu\left(t_{0}\right)\right)-f_{0}\left(\Upsilon(t), v_{\|}(t), \mu\left(t_{0}\right)\right)$. Details of this scheme, called direct $\delta f$, are given in Refs. [26] and [25].

\subsection{Quasi-neutrality equation}

Using the usual quasi-neutrality constraint, the Poisson equation is $n_{e}=Z_{i} n_{i}$. The polarization density, Eq. (5), contained in $n_{i}(\vec{x}, t)$ is simplified by using a long wavelength approximation $\left(k_{\perp} \rho_{L, i}\right)^{2} \ll 1$. The electron density $n_{e}(\vec{x}, t)$ is evaluated by assuming adiabatic (or Boltzmann) electrons along the magnetic field lines. After linearization of the ion polarization density the quasi- 
neutrality equation becomes

$$
\begin{aligned}
n_{e 0}(\psi) & +\frac{e n_{e 0}(\psi)}{T_{e}(\psi)}(\phi(\vec{x}, t)-\bar{\phi}(\psi, t)) \\
& =Z_{i}\left\langle n_{i 0}\right\rangle(\vec{x})+Z_{i} \vec{\nabla}_{\perp} \cdot\left(\frac{n_{i 0}(\psi)}{B \Omega_{i}} \vec{\nabla}_{\perp} \phi(\vec{x}, t)\right)+Z_{i} \delta n_{i}
\end{aligned}
$$

where $\langle\ldots\rangle$ is the average over the gyroangle,

$$
\begin{aligned}
\left\langle n_{i 0}\right\rangle(\vec{x})= & \int f_{0}\left(\Gamma\left(\psi(\vec{x}), \epsilon\left(v_{\|}, \mu, \vec{x}\right), \mu\right), \epsilon\left(v_{\|}, \mu, \vec{x}\right), \mu\right) \\
& \delta\left(\vec{R}+\vec{\rho}_{L, i}-\vec{x}\right) B_{\|}^{*} \mathrm{~d} \vec{R} \mathrm{~d} v_{\|} \mathrm{d} \mu \mathrm{d} \alpha,
\end{aligned}
$$

and

$$
\delta n_{i}=\int B_{\|}^{*} \mathrm{~d} \vec{R} \mathrm{~d} v_{\|} \mathrm{d} \mu \mathrm{d} \alpha \delta f\left(\vec{R}, v_{\|}, \mu, t\right) \delta\left(\vec{R}+\vec{\rho}_{L, i}-\vec{x}\right) .
$$

At this point, it is assumed that $\left\langle n_{i 0}\right\rangle(\vec{x})=n_{i 0}(\vec{x})$ and $n_{i 0}(\vec{x})=n_{e 0}(\psi) / Z_{i}$ for a local Maxwellian only. Nevertheless, in this paper it is assumed that this relation holds for any type of equilibrium distribution function, so $n_{i 0}(\vec{x})=$ $n_{e 0}(\psi) / Z_{i} \equiv n_{0}(\psi)$. The final quasi-neutrality equation is therefore:

$$
\frac{e Z_{i} n_{0}(\psi)}{T_{e}(\psi)}(\phi(\vec{x}, t)-\bar{\phi}(\psi, t))-\vec{\nabla}_{\perp} \cdot\left(\frac{Z_{i} n_{0}(\vec{x})}{B \Omega_{i}} \vec{\nabla}_{\perp} \phi(\vec{x}, t)\right)=Z_{i} \delta n_{i} .
$$

In Eq. (15), $\bar{\phi}$ is the flux-surface averaged potential. This term is nonzero only for axisymmetric perturbations: only the toroidal Fourier component $n=0$ gives a contribution to this term. The $n=0, m=0$ mode is commonly called the zonal flow.

\subsection{Particle and energy conservation}

Despite all the approximations made in the previous sections, the model derived from [9] still conserves the particle number and the energy (see [22]). The kinetic energy of the plasma is

$$
E_{\text {kin }}=\int m_{i}\left(\mu B+\frac{v_{\|}^{2}}{2}\right) f B_{\|}^{*} \mathrm{~d} \vec{R} \mathrm{~d} v_{\|} \mathrm{d} \mu \mathrm{d} \alpha .
$$

Its time derivative is

$$
\frac{\mathrm{d} E_{\text {kin }}}{\mathrm{d} t}=q_{i} \int \frac{\mathrm{d} \vec{R}}{\mathrm{~d} t} \cdot\langle\vec{E}\rangle f B_{\|}^{*} \mathrm{~d} \vec{R} \mathrm{~d} v_{\|} \mathrm{d} \mu \mathrm{d} \alpha
$$


In this model, the electrostatic energy can be defined as

$$
E_{\mathrm{f}}=\frac{q_{i}}{2} \int \mathrm{d} \vec{x}\left(\left\langle n_{i}\right\rangle(\vec{x}, t)-n_{0}(\vec{x})\right) \phi(\vec{x}, t) .
$$

The energy and particle number conservation are written:

$$
\begin{aligned}
& \frac{\mathrm{d} E_{\text {kin }}}{\mathrm{d} t}=-\frac{\mathrm{d} E_{\mathrm{f}}}{\mathrm{d} t} \\
& \frac{\mathrm{d} N_{\mathrm{ph}}}{\mathrm{d} t}=\frac{\mathrm{d}}{\mathrm{d} t} \int f B_{\|}^{*} \mathrm{~d} \vec{R} \mathrm{~d} v_{\|} \mathrm{d} \mu \mathrm{d} \alpha=0 .
\end{aligned}
$$




\section{$3 \quad$ Numerical implementation}

This section describes the implementation of the gyrokinetic model exposed in section 2. The general scheme of the code is shown in Fig. 1.

\section{$3.1 \delta f$ discretization: the Particle-In-Cell method}

The Particle-In-Cell (PIC) method [27] is commonly employed in gyrokinetic simulations $[8,16,17,22,28]$. The perturbed distribution function is discretized in the $5 \mathrm{D}$ phase space along trajectories with $N$ markers (also called tracers), carrying a weight $w$. In ORB $5, \delta f$ is discretized as:

$$
\delta f=\frac{N_{\mathrm{ph}}}{N} \sum_{p=1}^{N} \frac{1}{2 \pi B_{\|}^{*}} w_{p}(t) \delta\left(\vec{R}-\vec{R}_{p}(t)\right) \delta\left(v_{\|}-v_{\|, p}(t)\right) \delta\left(\mu-\mu_{p}\left(t_{0}\right)\right),
$$

where $N_{p h}$ is the number of physical particles. Each marker is characterized by its weight $w_{p}(t)$ and by its location $\left(\vec{R}_{p}(t), v_{\|, p}(t), \mu_{p}\left(t_{0}\right)\right)$ in the phase space. Integrating Eq. (21) over a small volume $\Omega_{p}$, the temporal evolution of $\delta f$ is obtained by solving the Vlasov equation for each weight $w_{p}(t)$ :

$$
\frac{\mathrm{d} w_{p}(t)}{\mathrm{d} t}=\frac{N}{N_{\mathrm{ph}}} \tau(\vec{E})_{p} \Omega_{p}
$$

with $\tau(\vec{E})$ given by equation (10), $\Omega_{p}=B_{\|}^{*} \mathrm{~d} \vec{R} \mathrm{~d} v_{\|} \mathrm{d} \mu \mathrm{d} \alpha / \mathrm{d} N$ represents the volume occupied by one marker in phase space and $\mathrm{d} N$ is the number of markers in an infinitesimal volume of phase space:

$$
\mathrm{d} N=\frac{N}{N_{\mathrm{ph}}} f_{L}\left(\vec{R}, v_{\|}, v_{\perp}\right) J(\chi, s, \varphi) \mathrm{d} s \mathrm{~d} \chi \mathrm{d} \varphi v_{\perp} \mathrm{d} v_{\|} \mathrm{d} v_{\perp} \mathrm{d} \alpha .
$$

The markers' loading is specified by the probability density function $f_{L}\left(\vec{R}, v_{\|}, v_{\perp}\right)$. The conventional loading is such that markers have a distribution function $p(s)=1-f_{g}+f_{g} \exp \left(\left(s-s_{0, L}\right)^{2} / \Delta s_{L}^{2}\right)$ in space, where $s_{0, L}$ and $\Delta s_{L}$ are input parameters, and are uniformly loaded in $\left(v_{\|}, v_{\perp}\right)$. A cut-off is applied at $\left(v_{\|}, v_{\perp}\right)=\kappa_{v} v_{\mathrm{th}, i}(s)$, where $\kappa_{v}$ is specified on input. During a simulation, the markers' distribution in the $(s,|v|)$ plane is stored for different times. This information is further used to build a new $f_{L}\left(\vec{R}, v_{\|}, v_{\perp}\right)$. This method is called optimized loading [22], and is aimed to minimize the statistical variance of the weights during the nonlinear phase of a second simulation which uses the new $f_{L}\left(\vec{R}, v_{\|}, v_{\perp}\right)$. Two different ways of initializing the weights (or equivalently the perturbation $\delta f$ ) have been implemented. The white noise 
initialization is defined by:

$$
\delta f_{p}\left(t_{0}\right)=A_{p} f_{0}\left(\vec{R}_{p}\left(t_{0}\right), v_{\|, p}\left(t_{0}\right), v_{\perp, p}\left(t_{0}\right)\right) \Omega_{p}
$$

$A_{p}$ is a pseudo-random number obtained with a Hammersley sequence, whose maximum amplitude is given on input. This scheme has the disadvantage that the initial perturbation is inversely proportional to the number of markers in the simulation. The simulation takes an increasing time, roughly proportional to the number of markers, until the physical modes emerge of the initial noise. Instead, the idea is to build a physical initialization, called mode initialization, independent of the number of markers:

$$
\begin{aligned}
\delta f_{p}\left(t_{0}\right) & =\Omega_{p} \frac{A_{0} f_{0}\left(\vec{R}_{p}\left(t_{0}\right), v_{\|, p}\left(t_{0}\right), \mu_{p}\left(t_{0}\right)\right)}{\left(m_{2}-m_{1}+1\right)\left(n_{2}-n_{1}+1\right)}\left|\frac{T\left(s_{0}\right)}{\vec{\nabla} T\left(s_{0}\right)}\right|\left|\frac{\vec{\nabla} T\left(s_{p}\left(t_{0}\right)\right)}{T\left(s_{p}\left(t_{0}\right)\right)}\right| \\
& \times \sum_{m=m_{1}}^{m_{2}} \sum_{n=n_{1}}^{n_{2}} \cos \left(m \chi_{p}\left(t_{0}\right)-n \varphi_{p}\left(t_{0}\right)\right)
\end{aligned}
$$

where $m_{0}, m_{1}, m_{2}, n_{0}, n_{1}, n_{2}$ are input parameters. This initialization will be stronger by choosing $m_{0}=\left[-n q\left(s_{0}\right)\right]$, where [...] denotes the integer part, as ITG modes are aligned with the field lines. It has the advantage that the initial perturbation converges with the number of markers and the initial phase of the simulation, until the physical modes develop, is independent of the number of markers.

\subsection{Equations of motion}

Tracers can be pushed either in cylindrical coordinates $(r, \varphi, z)$ or in magnetic coordinates $\left(\theta_{*}, s, \varphi\right)$. In order to avoid the singularity at the magnetic axis that would appear in $\mathrm{d} \theta_{*} / \mathrm{d} t$, it is more adequate to use $(\xi, \eta, \varphi)=$ $\left(s \cos \theta_{*}, s \sin \theta_{*}, \varphi\right)$. Among the numerous advantages of using magnetic coordinates, this set of coordinates avoids numerical interpolations during the construction of the perturbed density. Depending on the computer architecture, the simulation can be speeded up by $40 \%$. For both ad hoc and MHD equilibria, equilibrium coefficients needed for the pushing are obtained with linear interpolations from a $(r, z)$ or a $\left(s, \theta_{*}\right)$ grid.

For the integration of Eqs. (7), (8) and (22), a Runge Kutta integrator of order four is implemented in ORB5.

When a particle leaves the plasma, or equivalently when $s>1$, it is reflected: $\chi \rightarrow-\chi$. This scheme may lead to a small violation of unperturbed conserved quantities for equilibria that are not up-down symmetric.

The particle pushing is speeded up (up to 40\%) by using a cache sorting algorithm: particles are sorted in the poloidal plane every $n_{C S}$ time steps, where 
$n_{C S}$ is given on input.

\subsection{Discretisation of quasi-neutrality equation}

The quasi-neutrality equation (15) is solved with linear, quadratic or cubic B-splines finite elements [30]. The perturbed potential is discretized as:

$$
\phi(\vec{x}, t)=\sum_{\mu} \phi_{\mu}(t) \Lambda_{\mu}(\vec{x})
$$

where $\mu$ stands for $(j, k, l),\left\{\phi_{\mu}(t)\right\}$ are coefficients and $\left\{\Lambda_{\mu}(\vec{x})\right\}$ are tensor products of $1 D$ B-splines of order $p, \Lambda_{\mu}(\vec{x})=\Lambda_{j}^{p}(s) \Lambda_{k}^{p}(\chi) \Lambda_{l}^{p}(\varphi)$. The B-splines are defined on a $\left(N_{s}, N_{\chi}, N_{\varphi}\right)$ grid. To get a linear system for $\left\{\phi_{\mu}(t)\right\}$, the Galerkin method [31] is used. The system is then:

$$
\sum_{\mu} A_{\mu \nu} \phi_{\mu}(t)=b_{\nu}(t)
$$

with:

$$
\begin{aligned}
A_{\mu \nu} & =\int \mathrm{d} \vec{x} \frac{n_{0}(\psi)}{Z_{i} T_{e}(\psi)}\left(\Lambda_{\mu}(\vec{x}) \Lambda_{\nu}(\vec{x})-\bar{\Lambda}_{\mu}(s) \bar{\Lambda}_{\nu}(s)\right)+\frac{n_{0}(\psi)}{B \Omega_{i}} \vec{\nabla}_{\perp} \Lambda_{\mu}(\vec{x}) \cdot \vec{\nabla}_{\perp} \Lambda_{\nu}(\vec{x}) \\
b_{\nu}(t) & =\frac{N_{p h}}{N} \sum_{p=1}^{N} \frac{w_{p}(t)}{2 \pi} \int_{0}^{2 \pi} \mathrm{d} \alpha \Lambda_{\nu}\left(\vec{R}_{p}+\vec{\rho}_{L, i, p}(\alpha)\right)
\end{aligned}
$$

Note that the matrix is real, symmetric and positive definite. The building of $b_{\nu}(\vec{x}, t)$ is called the charge assignment. This projection onto the finite element basis is the main source of numerical noise. It becomes obvious that higher order splines are benefical for the noise reduction, as they have a more extended shape. The perpendicular gradients are approximated to lie in the poloidal plane, $\vec{\nabla}_{\perp} \cong \vec{\nabla}_{\text {pol }}=\vec{\nabla} s \frac{\partial}{\partial s}+\vec{\nabla} \chi \frac{\partial}{\partial \chi}$. Due to the axisymmetry in the toroidal direction, the system can furthermore be decoupled in $\varphi$. Applying a discrete Fourier transform on $\phi_{\mu}$ and $b_{\nu}$, and inserting these relations in (27) yields, in Fourier space:

$$
\sum_{\mu} A_{\mu \nu} \hat{\phi}_{\mu}^{(n)}(t)=\frac{\hat{b}_{\nu}^{(n)}(t)}{M^{(n), p}},
$$

where $(\mu, \nu)$ now stand for $2 D$ indices, $\hat{\phi}_{\mu}^{(n)}, \hat{b}_{\nu}^{(n)}(t)$ are Fourier coefficients of $\phi_{\mu}$ and $b_{\nu}$, and $M^{(n), p}$ is defined by:

$$
\sum_{l^{\prime}=1}^{N_{\varphi}} \int \mathrm{d} \varphi \Lambda_{l^{\prime}}^{p}(\varphi) \Lambda_{l}^{p}(\varphi) \exp \left(\frac{2 \pi i}{N_{\varphi}} n\left(l^{\prime}-l\right)\right)=M^{(n), p} .
$$


$M^{(n), p}$ can be computed analytically for any spline order $p$. The matrix $A_{\mu \nu}$ and the right hand side $b_{\nu}$ defined in Eq. (28) are modified with the Dirichlet boundary conditions $\phi(s=1, \chi, \varphi, t)=0$ and the regularity condition $\phi(s=0, \chi, \varphi, t)=\phi(s=0, \chi=0, \varphi, t)$. The matrix defined in Eq. (28) can be decomposed as $A_{\mu \nu}(\vec{x})=A_{\mu \nu}^{\text {lwa }}+A_{\mu \nu}^{\mathrm{ZF}}$, where $A_{\mu \nu}^{\mathrm{ZF}}$ contains the flux surface averaged terms only. The linear system associated with $A_{\mu \nu}^{\text {lwa }}$ can be solved with a sparse iterative solver, using the SSOR-preconditionned conjugate gradient method. It is not adequate for $A_{\mu \nu}^{\mathrm{ZF}}$ because its band is full. Another alternative is a direct solver using LAPACK routines. Although faster, it requires more memory. The memory needed to store the matrix becomes rapidly prohibitive as the grid size is increased. To reduce the memory storage, a parallel solver using SCALPACK routines has been implemented in ORB5, giving a reduction factor up to $N_{s} / 8$ for the memory of the matrix.

\subsection{Gyro-averaging}

The integral over the gyration angle for both electric field and perturbed density is computed with a $N_{g}=\min \left(\left(32, \max \left(4,4 \rho_{L, i, p} / \rho_{L, i}\right)\right)\right.$ discrete sum, where $\rho_{L, i, p}=v_{\perp, p} / \Omega_{i, p}$ is the marker gyroradius and $\Omega_{i, p}$ is the cyclotron frequency of the marker. Although it has been shown that a 4-points discretization is sufficient to describe perturbations up to $k_{\perp} \rho_{L, i} \sim 1$ [28], a gyro-adaptative method reduces noise since it acts like a Bessel filter [22]. The position $\left(r_{\alpha}, z_{\alpha}\right)$ of the marker on the Larmor ring is simply:

$$
\begin{aligned}
& r_{\alpha}=r_{G C}+\rho_{L, i, p} \cos \alpha \\
& z_{\alpha}=z_{G C}+\rho_{L, i, p} \sin \alpha
\end{aligned}
$$

When orbits are evolved in magnetic coordinates, the position of a marker is obtained by a Taylor expansion:

$$
\begin{aligned}
& \xi_{\alpha} \cong \xi_{G C}+\left.\vec{\rho}_{L, i, p} \cdot \vec{\nabla} \xi\right|_{G C} \\
& \eta_{\alpha} \cong \eta_{G C}+\left.\vec{\rho}_{L, i, p} \cdot \vec{\nabla} \eta\right|_{G C}
\end{aligned}
$$

Where:

$$
\vec{\rho}_{L, i, p}=\rho_{L, i, p}\left(\frac{\vec{\nabla} s}{|\vec{\nabla} s|} \cos \alpha+\frac{\vec{B} \times \vec{\nabla} s}{|\vec{B} \times \vec{\nabla} s|} \sin \alpha\right)
$$

This implementation has been successfully applied in [32] for the linear gyrokinetic code LORB5 [33]. 
Using Eqs. (4) and (26), the gyro-averaged electric field is given by:

$$
\langle\vec{E}\rangle=-\frac{1}{2 \pi} \sum_{\mu} \phi_{\mu}(t) \int \mathrm{d} \alpha \vec{\nabla} \Lambda_{\mu}\left(\vec{R}+\vec{\rho}_{L, i}(\alpha)\right)
$$

To achieve the best energy conservation, it is important to employ exactly the same gyro-averaging procedure for the charge assignment and the electric field [22].

\subsection{Fourier filter}

In order to reduce the noise, we apply a Fourier filter on the discretized perturbed density.

$$
F b_{i, j, k}=\sum_{m, n} f_{i, m, n} \widehat{b}_{i, m, n}(t) e^{i m \chi} e^{i n \varphi}
$$

A Fourier filter is of great interest since physically non relevant modes can be removed from the simulation. The poloidal Fourier filter should be chosen wide enough so as to include all modes relevant to the physics problem at hand, although a formal proof of energy conservation including Fourier filters cannot be done in toroidal geometry. One of the simplest filter, called square filter, is obtained by suppressing modes outside a window $\left[n_{\min }: n_{\max }\right] \times\left[m_{\min }: m_{\max }\right]$ specified on input. However, this kind of filter is inconsistent with the gyrokinetic ordering, as it retains modes with $k_{\|} / k_{\perp}$ much bigger than $\rho^{*}$ [34]. Indeed, ITG modes tend to align with the field lines. Mathematically, it means that these modes are such that $k_{\|}=(q(s) r)^{-1}(m+n q(s)) \rightarrow \rho^{*}$, consistent with the gyrokinetic ordering (2). So $m$ should be near $-n q(s)$. Toroidal-ITG modes are composed of a superposition of a few $m$ components around $m=-n q(s)$, resulting in $k_{\|} \approx(q(s) r)^{-1}$. In both cases, it is enough to keep a narrow window of modes in order to describe all physically relevant modes. The idea here is to define a surface-dependent filter that suppresses high $k_{\|}$modes. For each $n \in\left[n_{\min }: n_{\max }\right]$, the poloidal modes $[-n q(s) \pm \Delta m] \cap\left[m_{\min }: m_{\max }\right]$, where $\Delta m$ is an input parameter, are retained. It is very useful to use $\theta_{*}$ as the poloidal coordinate: the poloidal width of the spectrum for a toroidal mode is narrower with the straight-fieldline coordinate than with the geometrical poloidal angle $\theta$. In addition, when $\theta$ differs from $\theta_{*}$ (in case of elongation, triangularity or low aspect ratio), the mode is not peaked around $m=n q(s)$. Finally, the width of a toroidal mode spectrum increases with the plasma size when $\theta$ is used, whereas it is in principle independent of plasma size with $\theta_{*}$. The beneficial influence of a small $\Delta m$ will be explained in section 5 . 


\subsection{Code Parallelization}

Gyrokinetic simulations are extremely CPU time and memory demanding. Therefore, ORB5 is massively parallelized with MPI routines. A decomposition concept called domain cloning $[35,36]$ is applied: $P=P_{C} P_{\varphi}$, where $P$ is the total number of processors, $P_{\varphi}$ is the number of domains in the $\varphi$ direction and each domain is cloned $P_{c}$ times. After each charge assignment, the perturbed density is summed over the clones. After each step in the particle pushing, the markers are sorted to their appropriate $\varphi$ domain. The parallel direct solver described above is using a third MPI communicator, usually but not necessarily taken identical to the clone communicator. This parallelization scheme offers great flexibility because it can be tuned to different types of parallel platform architectures.

\subsection{Relations between physical and numerical parameters and $\rho *$ scaling}

Depending on the physical case under study, the ORB5 numerical parameters should be set up as follows. The radial mesh resolution depends on the maximum $k_{\rho} \rho_{L, i}$ that should be resolved, where $k_{\rho}$ is the radial component of the wave number. With cubic B-spline finite elements there should be at least 3 points per wavelength. Thus

$$
N_{s}>\frac{3}{2 \pi}\left(k_{\rho} \rho_{L, i}\right)_{\max } \frac{a}{\rho_{L, i}} .
$$

Similarly, for a maximum $k_{\chi} \rho_{L, i}$ that should be resolved up to the magnetic surface $s_{\max }$, where $k_{\chi}$ is the poloidal component of the wave number, the poloidal mesh should be set to

$$
N_{\chi}>3 s_{\max }\left(k_{\chi} \rho_{L, i}\right)_{\max } \frac{a}{\rho_{L, i}} .
$$

Since the perturbations tend to be aligned with magnetic field lines the toroidal mesh should be chosen as

$$
N_{\varphi} \approx N_{\chi} / q(s)
$$

The time step should satisfy the following three requirements:

$$
\omega_{\|} \Delta t \ll 1, \omega_{*} \Delta t \ll 1, \omega_{\mathrm{ExB}} \Delta t \ll 1
$$

with $\omega_{\|}=k_{\|} v_{\|}, k_{\|}=(q(s) r)^{-1}(m+n q(s)), \omega_{*}=v_{\text {th }, i}(d \ln T / d \rho) k_{\chi} \rho_{L, i}$ and $\omega_{\mathrm{ExB}}=k_{\perp} v_{\mathrm{ExB}}$. There is a limiting value of $k_{\|}$beyond which the ITG modes get strongly Landau damped. An estimate can be obtained from a dispersion 
relation [37], and taking the limit $\eta_{i} \rightarrow \infty$ :

$$
\left|k_{\|}^{\lim }\right| r=(1 / 2)\left(r / L_{T, i}\right) k_{\chi} \rho_{L i}
$$

The field-aligned Fourier filter of width $\pm \Delta m$ gives $\left|k_{\|}\right|_{\max }=\Delta m / r q(s)$. In order to resolve the physically relevant modes up to $\left|k_{\|}\right|^{\text {lim }}$ the width of the field-aligned Fourier filter should be

$$
|\Delta m|>\frac{q(s)}{2} \frac{r}{L_{T, i}} k_{\chi} \rho_{L i} .
$$

It is worth mentioning that it does not scale with $a / \rho_{L, i}$, since the typical values of $k_{\chi} \rho_{L, i}$ present in ITG turbulence do not depend on $a / \rho_{L, i}$. When quasi-neutrality equation is solved with $\theta$, Eq. (43) is no longer a good estimation. Note also that $\Delta m$ should be large enough so that the filtered perturbed density does not have discontinuities across magnetic surfaces. Mathematically, it is expressed as $\Delta m>n q^{\prime}(s) / N_{s}$. Using Eqs. (38) and (39) with $\left(k_{\rho} \rho_{L, i}\right)_{\max }=\left(k_{\chi} \rho_{L, i}\right)_{\max }=s_{\max }=1$, the last condition can be written:

$$
\Delta m \gtrsim 2 \hat{s}
$$

where $\hat{s}=s q^{\prime}(s) / q(s)$ is the magnetic shear. The first criterion of Eq. (41) gives

$$
\Omega_{i} \Delta t \ll \frac{r}{a} \frac{a}{\rho_{L, i}} \frac{q(s)}{|\Delta m|}
$$

or, if $|\Delta m|$ is chosen as in Eq.(43),

$$
\Omega_{i} \Delta t \ll \frac{2\left(a / \rho_{L, i}\right)(r / a)}{\left(r / L_{T, i}\right) k_{\chi} \rho_{L, i}} .
$$

The second criterion gives

$$
\Omega_{i} \Delta t \ll \frac{\left(a / \rho_{L, i}\right)(r / a)}{\left(r / L_{T, i}\right) k_{\chi} \rho_{L, i}} .
$$

The third criterion can be expressed in terms of the Mach number $M=$ $v_{\mathrm{ExB}} / v_{\mathrm{th}, i}$ as

$$
\Omega_{i} \Delta t \ll \frac{1}{M k_{\perp} \rho_{L, i}} .
$$

For most cases of interest for ITG driven turbulence $M$ is found to be of the order of $10^{-2}$ and $k_{\perp} \rho_{L, i}$ is at maximum of order unity. Using local estimates for the time step, it appears that $\omega_{\|}$is the fastest frequency of the system with a square filter, independently of the plasma size. With a field-aligned filter, $k_{\|}$ scales with $\rho^{*}$ and so for large plasmas $\omega_{E \times B}$ becomes the fastest frequency. 
The time step can be increased by a factor 10 , hence the simulations are strongly shortened. More details can be found in [34].

\subsection{Density and temperature profiles}

A given profile $A=T_{i}, T_{e}$ or $n_{0}$ is defined by:

$$
\frac{1}{A} \frac{\mathrm{d} A}{\mathrm{~d} \tilde{\psi}}=-\frac{a}{L_{A}} \frac{\left(1-\cosh ^{-2}\left[\frac{s_{0}}{\Delta_{A}}\right]\right)}{\left[\cosh ^{-2}\left(\frac{s-s_{0}}{\Delta_{A}}\right)-\cosh ^{-2}\left(\frac{s_{0}}{\Delta_{A}}\right)\right]} .
$$

$a / L_{T, i}, a / L_{T, e}, a / L_{n}, \Delta_{T, i}, \Delta_{T, e}, \Delta_{n}$ and $s_{0}$ are input parameters. All the gradients are peaked at $s_{0}$, therefore ITG modes should develop around that magnetic surface. Temperature profiles are normalized by their value at $s_{0}$. The density is normalized with the volume averaged density. Fig. 2 show typical profiles used in ORB5. 


\section{Scalability}

All simulations have been performed on the IBM BlueGene/L and Pleiades clusters of the Ecole Polytechnique Fédérale de Lausanne. The former has 4096 PowerPC $700 \mathrm{MHz}$ bi-processor nodes. The cluster has two different modes. In the coprocessor mode (CO), one processor is used with $512 \mathrm{MB}$ memory. In the virtual node $(\mathrm{VN})$ mode, both processors are used but with only $256 \mathrm{MB}$ memory each. Simulations done on the Pleiades cluster used 32 $2.8 \mathrm{GHz}$ Pentium 4 processors with $2 \mathrm{~GB}$ memory.

To measure the scalability, short simulations were performed at fixed grid size $\left(N_{s}=128, N_{\theta^{*}}=256, N_{\varphi}=512\right.$ for the VN mode and $N_{s}=128, N_{\theta^{*}}=$ $256, N_{\varphi}=256$ for the CO mode). The SCALAPACK parallel solver with 16 clones is employed. Simulations with 512, 1024, 2048 and 4096 processors have been made by varying the number of processors in the $\varphi$ direction $P_{\varphi}$. For each number of processors, several simulations with different number of markers per processor have been done. Since the size of the matrix is fixed, the time dedicated to the solver (backsolve and Fourier transforms) should be proportional to the number of toroidal slices per processor. The time dedicated for the particles (pushing + charge assignment) is assumed to scale linearly with the number of markers per processor. Finally, the communication time is mainly due to the $\varphi$-partition of the markers. It is hard to give a simple estimate to the communication time, since it depends on the time step, the cluster architecture, and the ion temperature. For the largest simulation done (1.5M markers/processor), the communication time represents $10 \%$ of the total simulation time. Therefore, the time per iteration is modelled as:

$$
t_{\text {it }}=\underbrace{K_{s} \frac{N_{\varphi} P_{c}}{P}}_{\text {solver }}+\underbrace{K_{m} \frac{N}{P}}_{\text {markers }}+\underbrace{t_{\text {comm }}}_{\text {communication }},
$$

where $K_{s}$ and $K_{m}$ are constants to be determined. Fig. 3 displays the time per iteration $t_{\text {it }}$ as a function of the number of markers per processor $N / P$. The dependance is linear, as expected. However, the slope of these fits, namely $K_{m}$ seems to slowly increase with $P$, which illustrate a slight derivative from an ideal scaling. $K_{s}$ and $K_{m}$ have been obtained by a linear fit of $t_{\text {it }}$ as a function of $N / P$ at fixed $P=512$. Therefore, the measured time per iteration can be further compared to the fitted time $t_{\mathrm{it}}^{f}$. Fig. 4 shows the ratio $t_{\mathrm{it}} / t_{\mathrm{it}}^{f}$ as a function of $P$. The maximum value of the relative degradation due to the increase of the number of processors is only $15 \%$. Effects of $t_{\text {comm }}$ can be included by fitting $t_{\text {it }}-t_{\text {comm }}$ instead of $t_{\text {it }}$. In that case, the maximum relative degradation falls down to $10 \%$.

Globally, these results show the excellent scaling properties of ORB5 up to 4096 processors. 


\section{Simulations results}

\subsection{Field-aligned filter}

The field-aligned filter has been tested with the following input parameters: $m_{i}=1, a=40 \rho^{*}, B_{0}=1[T], R_{0}=1[m], R_{0} / a=5$. The density profile is flat, $T_{i}=T_{e}, R / L_{T, i}=12, \Delta_{T, i}=0.208, s_{0}=0.5 . \quad N=2^{24} \cong 16 \mathrm{M}$ markers, $\Delta t=40 \Omega_{i}^{-1}$. The initial distribution $f_{0}$ is a canonical maxwellian with correction, (see (9)). $\delta f$ is obtained through the conventional $\delta f$ scheme and is initialized with white noise. The quasi-neutrality equation is solved with cubic B-splines on a $N_{s}=128, N_{\theta^{*}}=128, N_{\varphi}=64$ grid with the parallel direct solver. The main parameter of the field-aligned filter is the width $\Delta m$. By keeping only small $k_{\|}$modes, the number of Fourier modes kept in the filter is strongly reduced. A square filter has $N_{s}\left(2 m_{\max }+1\right)\left(2 n_{\max }+1\right) \propto\left(\rho^{*}\right)^{-3}$ whereas a field-aligned filter has approximatively $N_{s}(2 \Delta m+1)\left(2 n_{\max }+1\right) \propto$ $\left(\rho^{*}\right)^{-2}$. The latter reduces the number of modes by about $\left(\rho^{*}\right)^{-1}$. In [38], it is shown that numerical noise, due to the projection of the charge density onto a finite number of markers, mainly depends on the square root of the number of markers per Fourier modes. In that sense, a field-aligned filter should improve the quality of a simulation without affecting CPU time, in contrary to an increase of the numbers of tracers. A good indicator of the quality of the simulation is the energy conservation described at section 2.5. Fig. 5 shows the relative energy conservation $\Delta E / E_{f}$, where $\Delta E=E_{\text {kin }}(t)+$ $E_{f}(t)-E_{\text {kin }}\left(t_{0}\right)-E_{f}\left(t_{0}\right)$ for simulations with square and field-aligned filters . In a noise-free simulation, or equivalently in the limit of an infinite number of markers and an infinitely small time step, $\Delta E / E_{f}$ should be zero. A gain of 2 orders of magnitude in the relative energy conservation is obtained as the filter goes from square to field-aligned. With a square filter, the energy deviation represents approximatively $1 \%$ of the initial energy of the plasma which is an unacceptable value. Fig. 6 displays the volume-averaged radial heat flux $Q$, defined by

$$
Q=\left.\frac{1}{V} \sum_{p=1}^{N} w_{p} \frac{1}{2} m v_{p}^{2} \frac{\langle\vec{E}\rangle \times \vec{B}}{B_{\|}^{*} B} \cdot \frac{\vec{\nabla} \psi}{|\vec{\nabla} \psi|}\right|_{\vec{R}_{p}, v_{\|, p}, \mu_{p}}
$$

where $V$ is the volume of the torus, for field-aligned and square simulations. In the latter case, a numerical heating develops in the late nonlinear phase. This kind of phenomenon is typical when too few markers are used. The square filter simulation would explode because of numerical noise if it was continued. This simple simulation already points out the link between the number of markers and the number of Fourier modes kept in the simulation. After the saturation, the plasma should reach a quasi-steady state. This property is obviously lost, as can be seen from Fig. 7 . However, for the field-aligned filter, 
the energy of $n \neq 0$ modes is constant for late times (see Fig. 8). On Fig. 9, the radially averaged energy spectrum of the $n=6$ mode in the poloidal space is shown at different times for a square filter simulation. In the linear phase, the mode is peaked near $m=-12=-n q\left(s_{0}\right)$ as $n=6$ and $q\left(s_{0}\right)=2$. After the saturation, the peak energy becomes smaller but the spectrum becomes much broader. On Fig. 10, the spectrum is normalized to the energy peak of the mode. The spectrum is no longer peaked and $m$ components which are far away from $n q\left(s_{0}\right)$ contain a very significant part of the total toroidal mode energy. The field energy of all $n \neq 0$ modes is growing in time but the peak energy decreases: this is a clear evidence that numerical noise is created because of high $k_{\|}$modes. It is of course suppressed by applying a field-aligned filter (see Fig. 8). In this case, the only growing mode is the zonal flow, which is not really affected by the parallel dynamics as it is strongly dominated by the $m=0$ component. Its energy increases mainly due to numerical noise, which is still kept in the field-aligned filter. The bad quality of the square filter can be observed in Fig. 11, which shows the electric potential on a magnetic surface. The resulting structure is clearly a superposition of high $k_{\|}$modes, whereas the field-aligned filter naturally preserves the field-aligned structure of ITG modes.

The determination of $\Delta m$ is a very important step. A too small value of $\Delta m$ will obviously cut some relevant physics, whereas a too large $\Delta m$ introduces additional numerical noise. The argument to fix $\Delta m$ is to converge the growth rate of toroidal modes in the linear phase. Fig. 12 shows the evolution of the mode $n=6$ for different values of $\Delta m$ and a square filter simulation. Small $\Delta m$ cases unsurprisingly yield lower growth rates. Convergence is reached with $\Delta m=5$. To summarize, smart Fourier filtering is a powerful numerical scheme to improve the quality of a PIC simulation: by relaxing the time step criterion and by decreasing the number of Fourier modes in the simulations, $\mathrm{CPU}$ time is reduced by 2 orders of magnitude. In addition, the field-aligned filter should be even more efficient in the limit of small $\rho^{*}$ plasmas.

\subsection{Convergence with number of markers}

In collisionless gyrokinetic simulations, convergence is a subtle notion. For PIC simulations, not only the time step and the grid resolution need to be carefully chosen, but the number of markers plays a crucial role as well. Indeed, numerical noise inherent to the PIC method may determine the level of transport in ETG simulations [39]. In ITG PIC simulations, due to the strong influence of the zonal flow, the situation is different, however the question of the required number of markers for convergence still remains. By measuring the level of numerical noise in ETG simulations, it has been established that the number of markers required is linked to the number of Fourier modes in the simulation [38]. A study of numerical noise in ITG simulations is beyond 
the scope of this paper. Here, the question of convergence is approached by means of physics diagnostics. Fig. 14 shows the evolution of the volume averaged heat flux for different numbers of markers. The overshoot is shifted in time, as the initial level of the perturbation is inversely proportional to $\sqrt{N}$. The sole Fig. 14 is not sufficient to say if a reasonable convergence is reached or not. This difficulty can be overcome by using the mode initialization with a single mode $\left(m_{0}, n_{0}\right)$ (see section 3.1). Fig. 13 shows again $Q$ for a marker scan performed with the mode initialization. As the number of tracers is increased, the different curves look more and more alike during the whole simulation. This gives confidence about the number of tracers needed for a converged simulation. From $32 M$ markers, all the heat flux curves are quite close to each other. Note that both initialization give quite different overshoots: for the mode initialization, a single toroidal mode strongly dominates since the beginning of the simulation, whereas all toroidal modes have approximatively the same initial energy when the white noise initialization is employed. In this context, a multiple mode initialization would be more appropriate, but in principle the convergence level of a simulation should not depend on the initialization.

\subsection{The Rosenbluth-Hinton test}

The test consists in comparing the numerical calculation of the time evolution of the axisymmetric potential with the analytical result, valid for circular magnetic surfaces in the limit of large aspect ratio, obtained by Hinton and Rosenbluth [40]. The gyrokinetic Vlasov equation for the zonal flow component, i.e. $n=m=0$, is analytically solved and the axisymmetric component of an electrostatic perturbation is found to linearly damp and a residual flow level is found. Therefore, the $E \times B$ velocity $\left(v_{\vec{E} \times \vec{B}}\right.$, normalized to the initial value $\left.v_{\vec{E} \times \vec{B}}(0)\right)$, generated by a pure axisymmetric density perturbation, is expected to behave as:

$$
\frac{v_{\vec{E} \times \vec{B}}}{v_{\vec{E} \times \vec{B}}(0)}=\left(1-A_{R_{0}}\right) e^{-\gamma_{G} t} \cos \left(\omega_{G} t\right)+A_{R_{0}}
$$

where $A_{R_{0}}$ is the residual, at the radial position $\rho$ :

$$
A_{R_{0}}=\frac{1}{\left(1+1.6 q(s)^{2}\left(\rho / R_{0}\right)^{-1 / 2}\right)}
$$


and $\omega_{G}, \gamma_{G}$ are respectively the frequency and the decaying rate of the velocity perturbation [41]:

$$
\begin{aligned}
\omega_{G} & =\left(1+\frac{1}{2 q(s)^{2}}\right)^{1 / 2} \frac{\sqrt{2} v_{\mathrm{th}, i}}{R_{0}} \\
\gamma_{G} & =\omega_{G} \exp \left(-q(s)^{2}-\frac{1}{2}\right)
\end{aligned}
$$

The expression for the frequency (54) was derived in [41]. An underestimation or overestimation of the residual zonal flow would lead to an incorrect prediction of the ion diffusivity $\chi_{i}$. Therefore a numerical code must provide the correct residual $A_{R_{0}}$ in order to produce reliable physical results.

The zonal flow damping test has been performed in linear mode, which means that nonlinear terms in particle trajectory equations have been suppressed. In order to reproduce the results of Hinton and Rosenbluth, we solve only for the $n=0, m=0$ component of the electrostatic potential, the other modes are Fourier filtered. Input parameters are $m_{i}=1, a=40 \rho^{*}, B_{0}=$ $1.44[T], R_{0}=5[\mathrm{~m}], R_{0} / a=10$. The density and temperature profiles are flat, $T_{i}=T_{e}$. The safety profile is monotonic and two different magnetic surfaces have been studied, $s_{0}=0.5, q\left(s_{0}\right)=1.15$ and $s_{0}=0.7, q\left(s_{0}\right)=1.33$. The grid is $N_{s}=64, N_{\chi}=64, N_{\varphi}=64$, the number of markers is $N=16 \mathrm{M}$, the time step is $\Delta t=50 \Omega_{i}^{-1}$ and a canonical Maxwellian with correction is used. The initial condition has been prepared in order to obtain an axisymmetric ion density perturbation. The results must not depend on the initial conditions, therefore two different perturbations of the ion density have been tested, $\delta n_{i}(s)=\delta n_{i, 0} \cos (\pi s)$ and $\delta n_{i}(s)=\delta n_{i, 0} \sin (\pi s)$, where $\delta n_{i, 0}$ is chosen

so that $\left\langle v_{\vec{E} \times \vec{B}}\right\rangle_{s}(t=0)=0.07 v_{\mathrm{th}, i}$. The results of the ORB5 simulations are plotted in Figs. 15 and 16. In these figures, the $E \times B$ velocity normalized at the initial value $v_{\vec{E} \times \vec{B}} / v_{\vec{E} \times \vec{B}}(0)$ is plotted as a function of time. As a reference, the residual evaluated from Eq. (53) and the exponential decay predicted by Eq. (55) are also plotted. In all cases the results are in good agreement with the residual predicted by Rosenbluth-Hinton theory. Table 1 gives a summary of the frequencies $\omega_{G}$ and decaying rates $\gamma_{G}$ from the simulations, compared to the values predicted by the theory. We find an overall good agreement between numerical results and theory predicted values.

\subsection{CYCLONE benchmark}

In Ref. [43], several fluid, gyrofluid and gyrokinetic codes are compared for the so called CYCLONE test case, which represents local parameters from an ITER-relevant DIII-D H-mode shot [42]. The paramters are $\rho^{*}=$ $1 / 175, a=0.48[\mathrm{~m}], B_{0}=1.91[T], R_{0}=1.32[\mathrm{~m}], s_{0}=0.624$ (corresponds to $\left.\rho_{0}=0.5 a\right), q\left(s_{0}\right)=1.4, T_{i}=T_{e}, R_{0} / L_{T, i}=6.9, \eta_{i}=L_{n} / L_{T, i}=3.12, \hat{s}=0.78$ 
$\Delta_{T, i}=0.3 . \quad N=2^{26} \cong 83 M$ markers, $\Delta t=40 \Omega_{i}^{-1}$. The quasi-neutrality equation is solved with cubic B-splines on a $N_{s}=128, N_{\chi}=448, N_{\varphi}=320$ grid and a field-aligned filter with $\Delta m=5$, is applied. The value of $\rho^{*}$ is the smallest possible having regard to the computational power. Benchmarking ORB5 for these parameters is crucial in order to have confidence in the code. A first simple test is presented on Fig. 17. ORB5 has been run in the linear mode. $\rho^{*}$ has been changed to $1 / 140$, a local maxwellian and the exact same equilibrium profiles have been employed in an effort to have similar parameters between the different codes. Both codes show excellent agreement.

The numerical quality of CYCLONE nonlinear simulation is shown through the relative energy conservation on Fig. 18. In the linear phase, the field energy is very small and the relative energy conservation can be arbitrarily high, but in the nonlinear phase relative energy conservation becomes a valid check. For this CYCLONE simulations, energy is conserved up to very long times within $30 \%$ of the field energy, which is a remarkable value for a global PIC code. This energy deviation represents $10^{-5}$ of the initial kinetic energy of the system. As the quasi-equilibrium state establishes, numerical noise grows and slowly leads to the loss of energy conservation. However, for late times the system is close to marginal stability because of profile relaxation so the state of the system will not provide any new physical information. Hence it is useless to continue a PIC collisionless simulation to very long times. Therefore a strong relative energy deviation for late times is not significant. The situation could be different if collisional sources were added to the simulation.

Nonlinear benchmark is usually performed by plotting the ion diffusivity defined by $\chi_{i} \equiv-Q /\left(n_{i} \vec{\nabla} T_{i}\right)$ versus $R_{0} / L_{T, i}$. Note that no assumption is done on $Q, n_{i}$ and $\vec{\nabla} T_{i}$ : these profiles are reconstructed with appropriate moments of Vlasov equation and then smoothed using splines with tension interpolation [44]. In [43], Dimits proposed a fit to express the ion diffusivity as a function of $R_{0} / L_{T, i}$ when the system has reached (quasi-)steady state:

$$
\frac{\chi_{i}}{\chi_{\text {Dimits }}} \cong 15.4\left(1-6 \frac{L_{T, i}}{R_{0}}\right)
$$

with $\chi_{\text {Dimits }}=\chi_{\mathrm{GB}} a / L_{n}$ where $\chi_{\mathrm{GB}}=\left(\rho^{*}\right)^{2} c_{s} / a$ is the gyro-Bohm transport coefficient [45]. There are two difficulties in benchmarking ORB5 against the Dimits fit, which has been obtained with a flux-tube code. Firstly, spatial averaging must be applied since ORB5 is a global code. Secondly, the lack of sources implies that the temperature profile is not frozen as in flux-tube codes and relaxes during the simulation. It is therefore much adequate to characterize the radial transport by a cloud of points $\left(\chi_{i}, R_{0} / L_{T, i}\right)$ representing the time evolution of space averaged values rather than with a single point. Such a procedure has been applied in [46]. ORB5 results are displayed in Fig. 19. At the begining of the simulation, the radial transport is null and the logarithmic gradient variation is very weak. Then the turbulence establishes, leading to profile relaxation. Finally, the system is in quasi-equilibrium state, 
meaning that the ion diffusivity decreases to 0 and the logarithmic gradient is close to marginal stability. The cloud of points is well located around the Dimits fit. The dispersion is more important for small averaging widths $\Delta s$. A large $\Delta s$ allows the reduction of global profile effects, which may lead to different predictions $[47,48]$, especially for the relatively large $\rho^{*}$ used in these ORB5 simulations. Local profiles are shown in Fig. 20. Remark how fast the profile relaxes to a quasi-equilibrium state. A way to prevent this phenomenon would be to decrease $\rho^{*}$, thus reducing global effects. Unfortunately simulations at lower $\rho^{*}$ were not possible because of the limited computational power. 


\section{Conclusion}

The global collisionless PIC code ORB5 is a powerful tool for microinstabilities studies. It relies on a gyrokinetic model which conserves energy and particle number, providing useful checks of the numerical solution at each time step. Based on the $\delta f$ method, this codes uses true equilibrium functions to prevent spurious zonal flows. Several noise reduction techniques are implemented, such as adaptative gyro-averaging, optimized loading and Fourier filtering. In this paper, it is shown that simulations based on field-aligned Fourier filtering combined with the use of straight-field line coordinates have lead to a speedup of 2 orders of magnitude. More precisely, the time step is relaxed by removing the high $k_{\|}$modes from the turbulence spectrum, which are anyhow inconsistent with the gyrokinetic ordering, and the number of markers needed in the simulation is strongly reduced because the simulation contains much less Fourier modes. By using a straight-field-line poloidal coordinate instead of the poloidal geometrical angle, the required width of the field-aligned filter is minimal and most importantly becomes independent of the plasma size. The latter remark is of considerable interest as future tokamaks such as ITER will have extremely small $\rho^{*}$. Magnetic coordinates and the field-aligned filter are therefore important steps towards the simulation of realistic devices. ORB5 also differs from other gyrokinetic codes as it evolves particle orbits in magnetic coordinates, therefore avoiding costly interpolations during charge assignment. ORB5 shows excellent scalability properties: a proper parallelization is crucial with the development of large scale computers. The question of convergence with the number of markers has been studied by applying a physical initialization of the perturbation instead of random noise. Finally, the code has been successfully benchmarked against other gyrokinetic codes. The next step will be the implementation of several relevant effects missing in the recent model, such as kinetic electron dynamics, collisions, impurities, electromagnetic effects, sources and sinks, in order to reduce the gap between experimental and theoretical transport predictions.

\section{Acknowledgements}

This work was partly supported by the Swiss National Science Foundation. Computations were performed on the BG/L and Pleiades clusters of the Ecole Polytechnique Fédérale de Lausanne. 
INPUT: PARTICLE LOADING

- UNIFORM

- SPECIFIED

- OPTIMIZED

\section{INPUT: $\delta$ f INITIALIZATION}

- white noise initialization

- mode initialization
INPUT: EQUILIBRIUM

- MHD (from CHEASE code)

- $\mathrm{AD} \mathrm{HOC}$

Initial distribution function:

- LOCAL Maxwellian

- CANONICAL Maxwellian (+ electron density integration)

Coordinate system:

- TOROIDAL coordinates $(\mathrm{s}, \theta, \varphi)$

- MAGNETIC FIELD LINES coordinates $\left(\mathrm{s}, \theta_{*}, \varphi\right)$

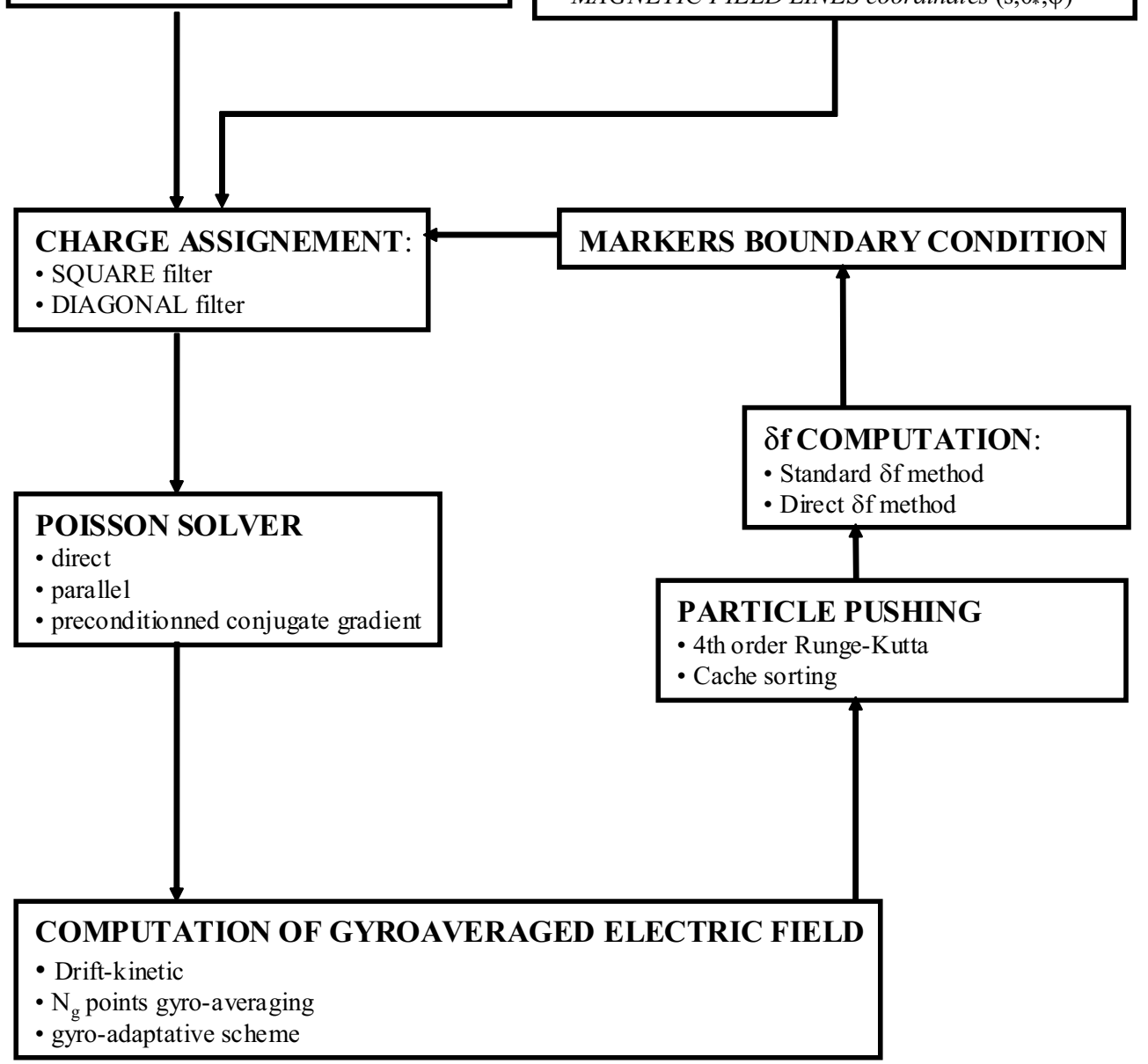

Figure 1. ORB5 scheme. 

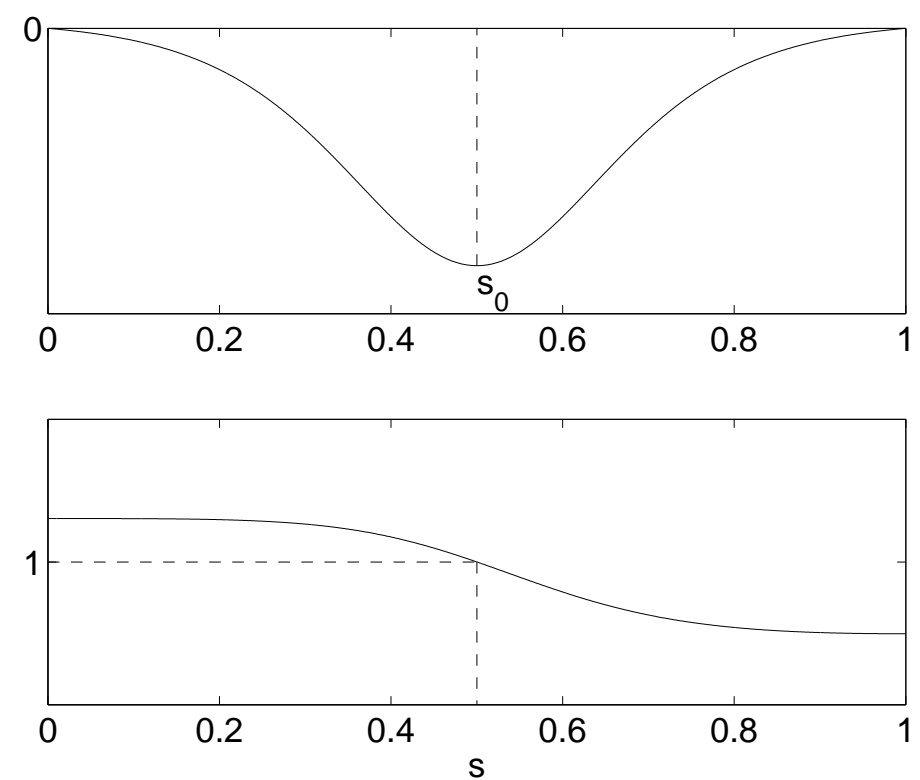

Figure 2. Logarithmic gradient (top) and profile (bottom) used in ORB5.
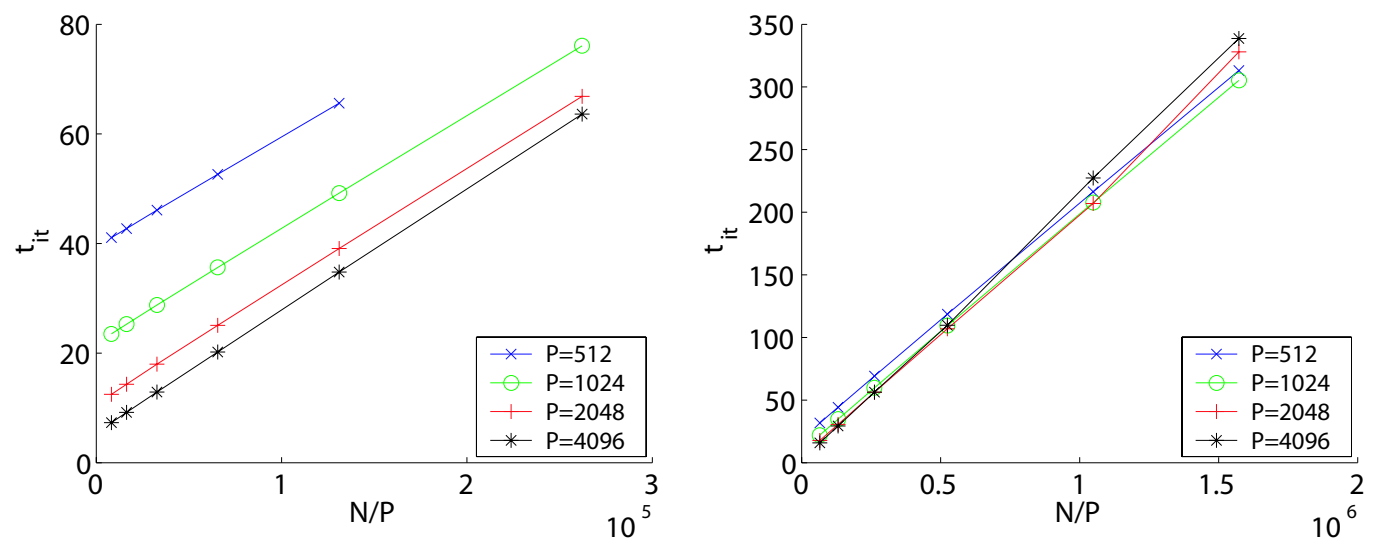

Figure 3. Time per iteration as a function of $N / P$ for the $\mathrm{VN}$ mode (left) and the $\mathrm{CO}$ mode (right) of $\mathrm{BG} / \mathrm{L}$. 

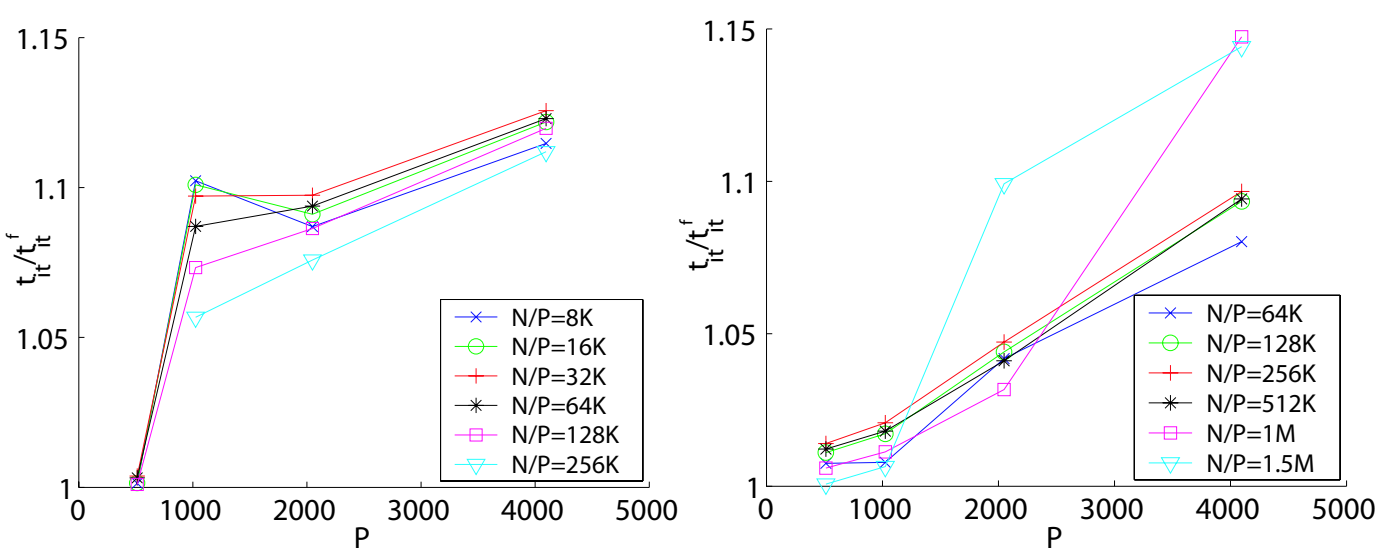

Figure 4. $t_{\mathrm{it}} / t_{\mathrm{it}}^{f}$ as a function of $P$ for the $\mathrm{VN}$ mode (left) and the CO mode (right) of $\mathrm{BG} / \mathrm{L}$.

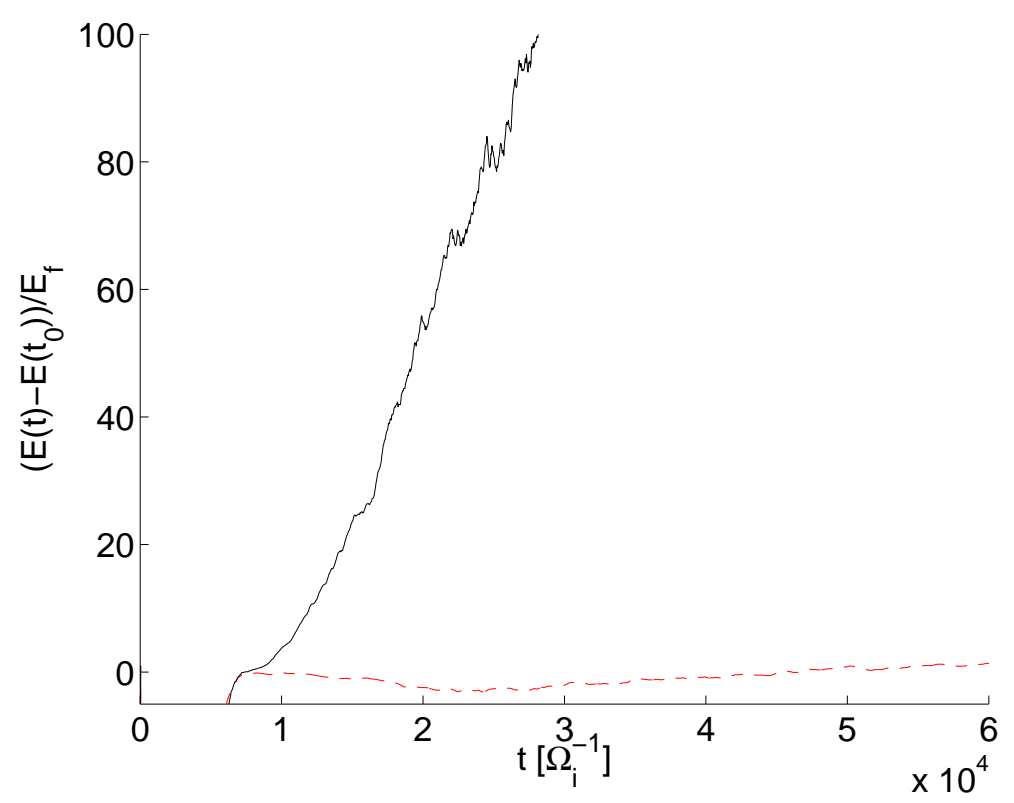

Figure 5. Relative energy conservation for a square filter simulation (black, solid) and a field-aligned simulation (red, dashed). 


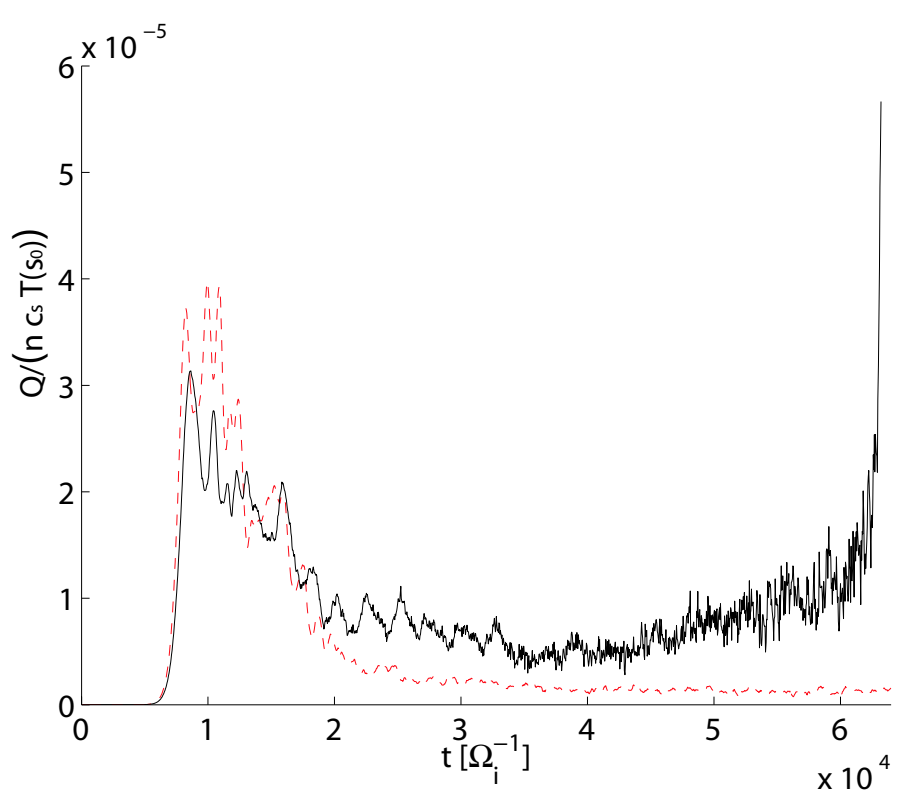

Figure 6. Volume averaged radial heat flux for a square filter simulation (black, solid) and a field-aligned simulation (red, dashed).

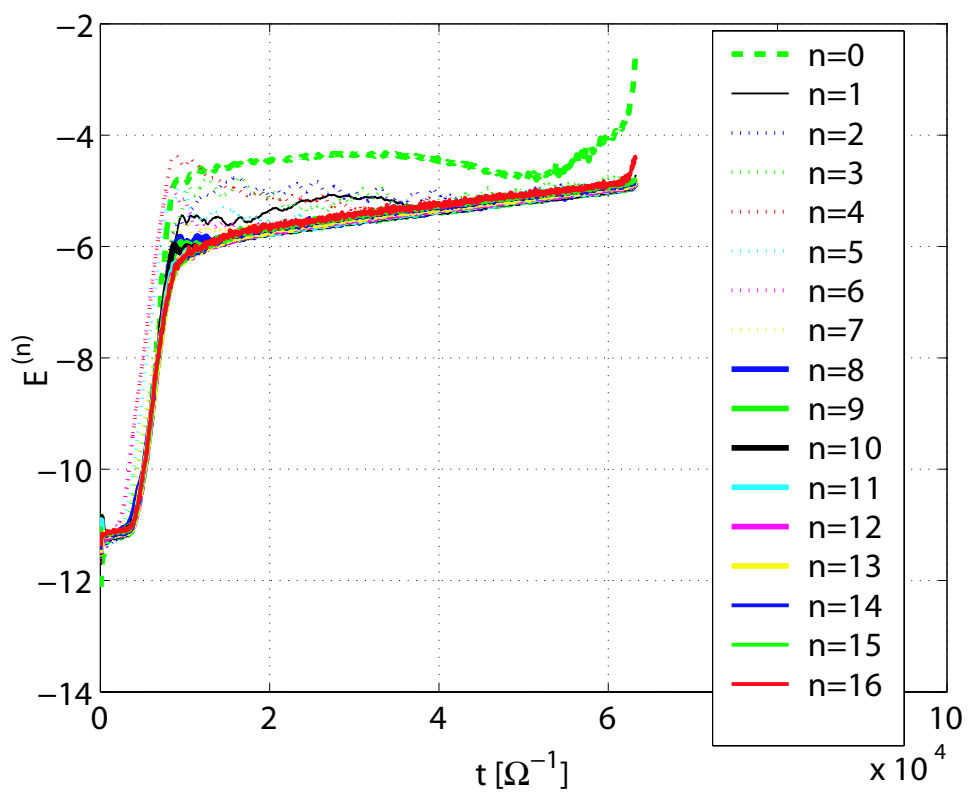

Figure 7. Energy of toroidal modes for a square filter simulation. Dashed line is the $n=0$ mode. 


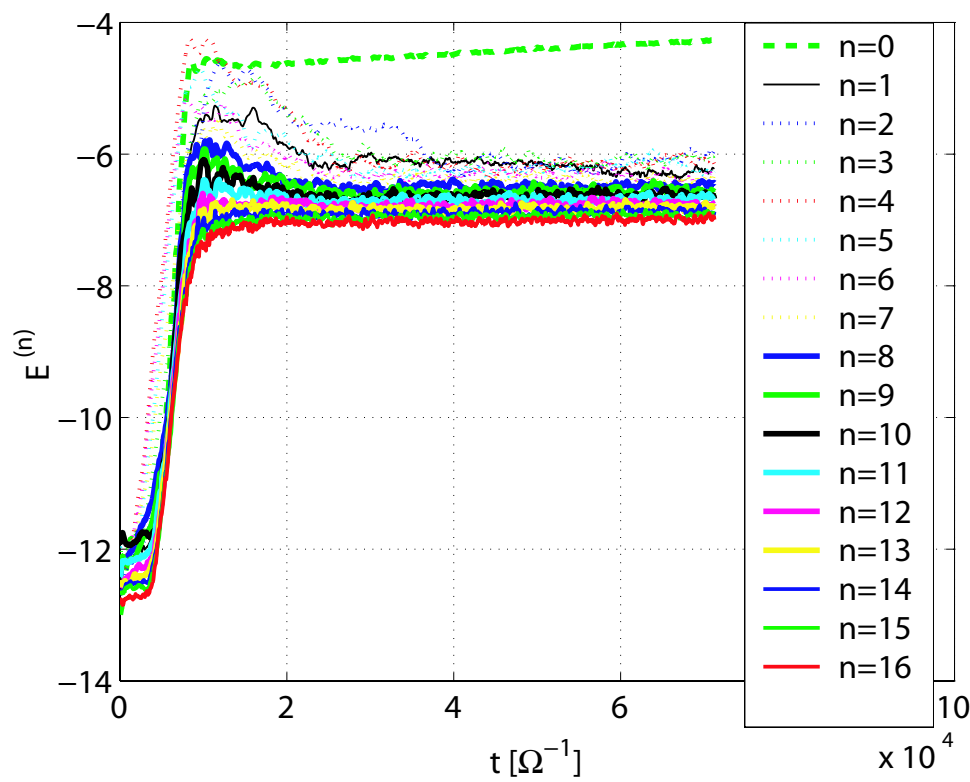

Figure 8. Energy of toroidal modes for a field-aligned filter simulation with $\Delta m=4$. Dashed line is the $n=0$ mode.

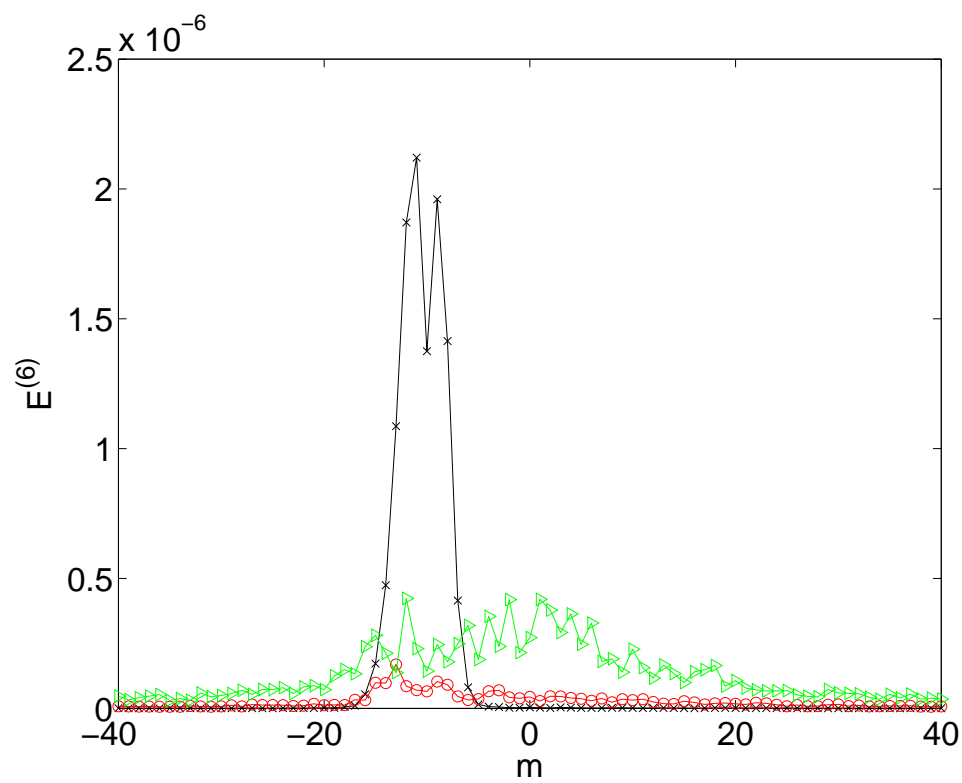

Figure 9. Poloidal spectrum of energy for toroidal mode $n=6$ at time $t=8000 \Omega_{i}^{-1}$ (linear phase, black, crosses), $t=24000 \Omega_{i}^{-1}$ (red, circles) and $t=48000 \Omega_{i}^{-1}$ (green, triangles) for a square filter simulation. 


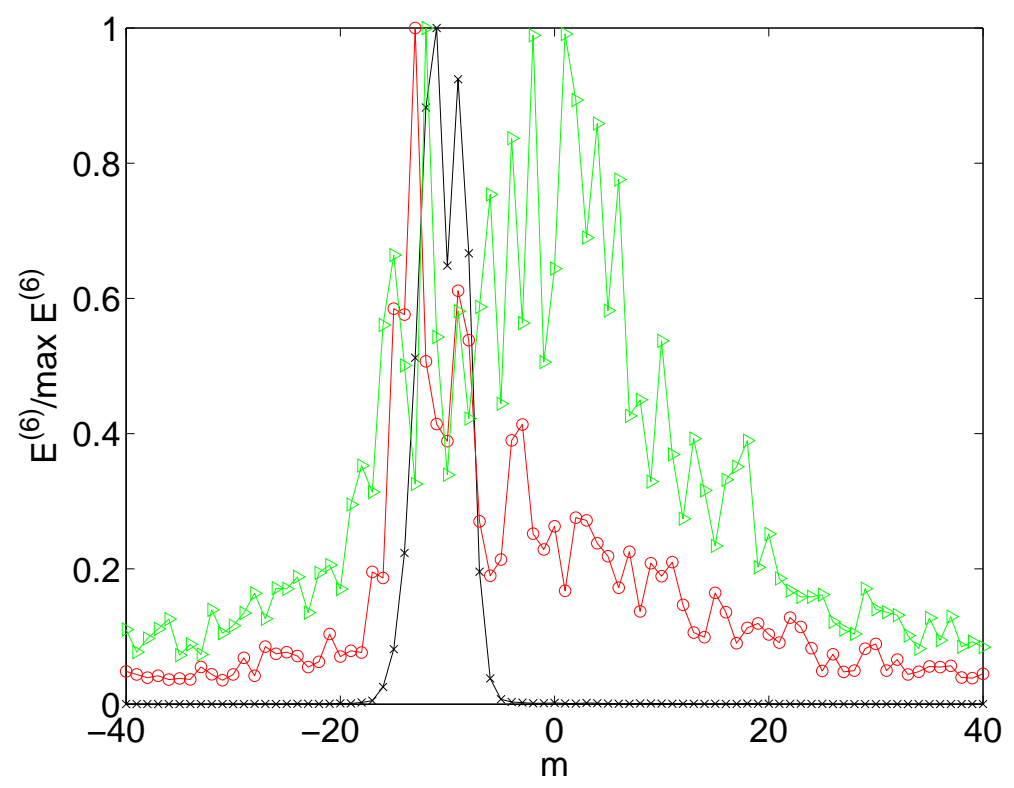

Figure 10. Poloidal spectrum of energy for toroidal mode $n=6$, normalised to the largest component, at time $t=8000 \Omega_{i}^{-1}$ (linear phase, black, crosses), $t=24000 \Omega_{i}^{-1}$ (red, circles) and $t=48000 \Omega_{i}^{-1}$ (green, triangles) for a square filter simulation.
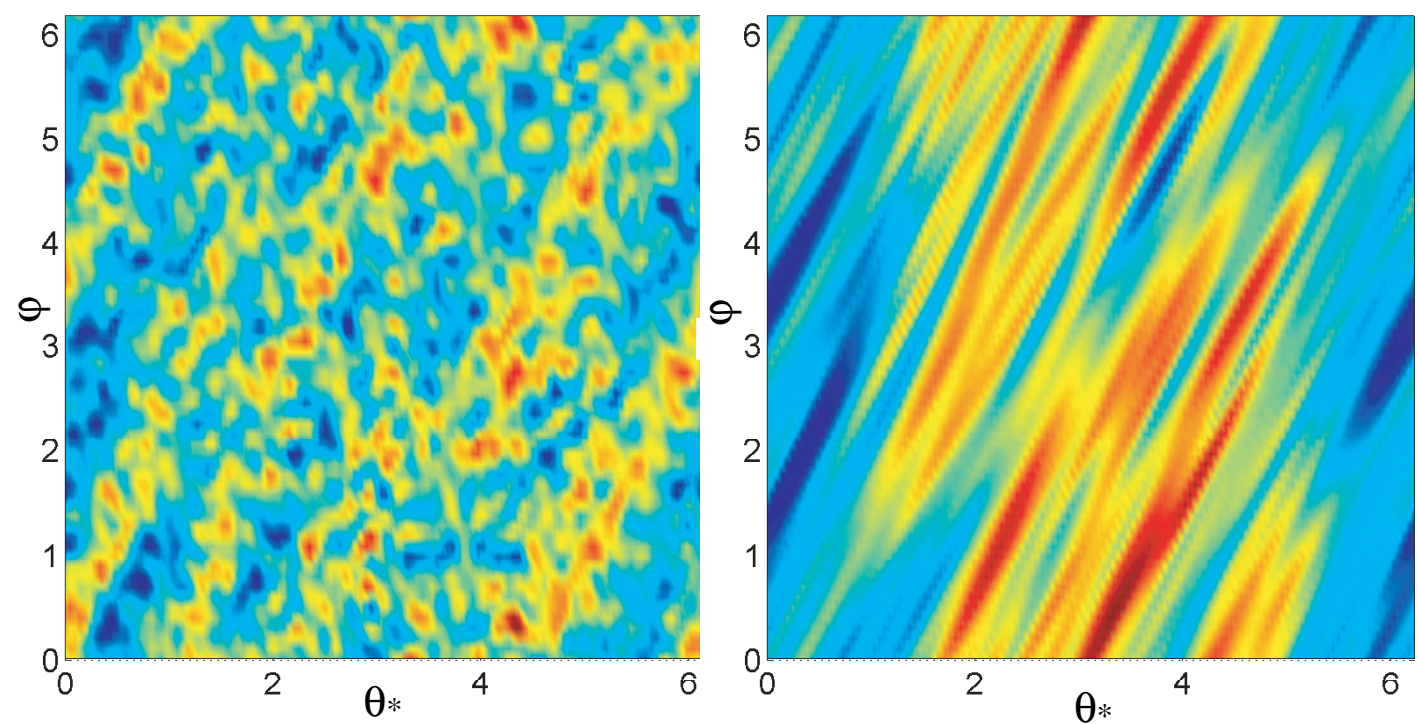

Figure 11. Perturbed electric potential along $s_{0}=0.5$ at $t=50000 \Omega_{i}^{-1}$ in the $\left(\theta_{*}, \varphi\right)$ plane for a square filter (left) and a diagonal filter with $\Delta m=4$ (right). 


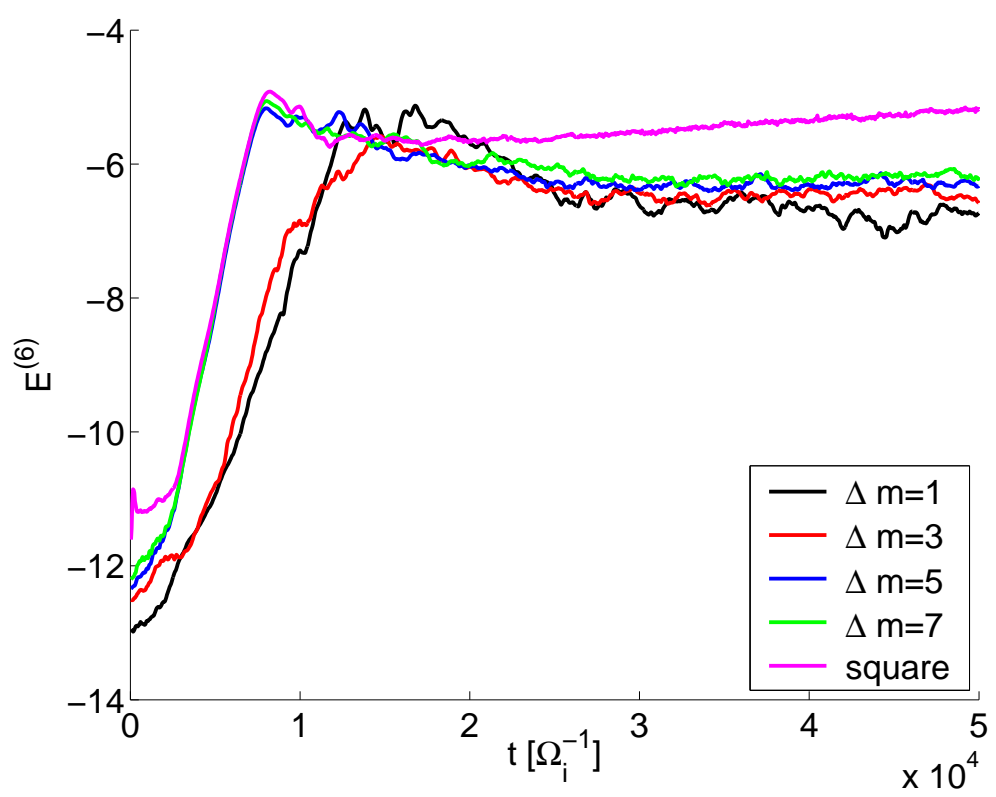

Figure 12. Energy of the $n=6$ mode for a field-aligned filter with different values of $\Delta m$ and a square filter.

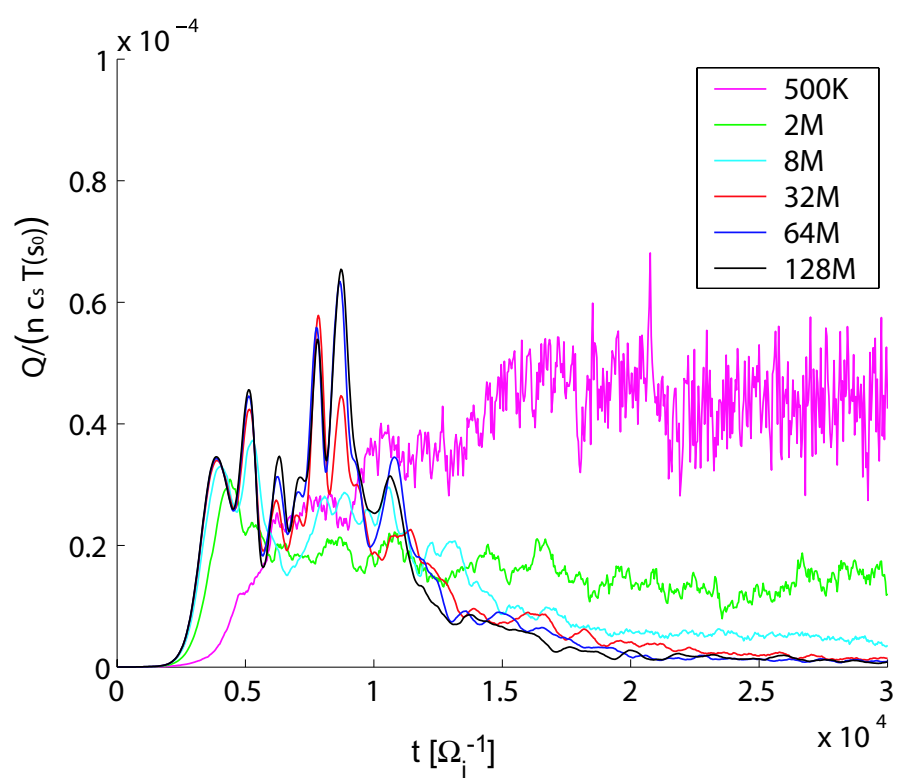

Figure 13. Time evolution of radial heat flux for different markers number with field-aligned filter and a single mode initialization. 


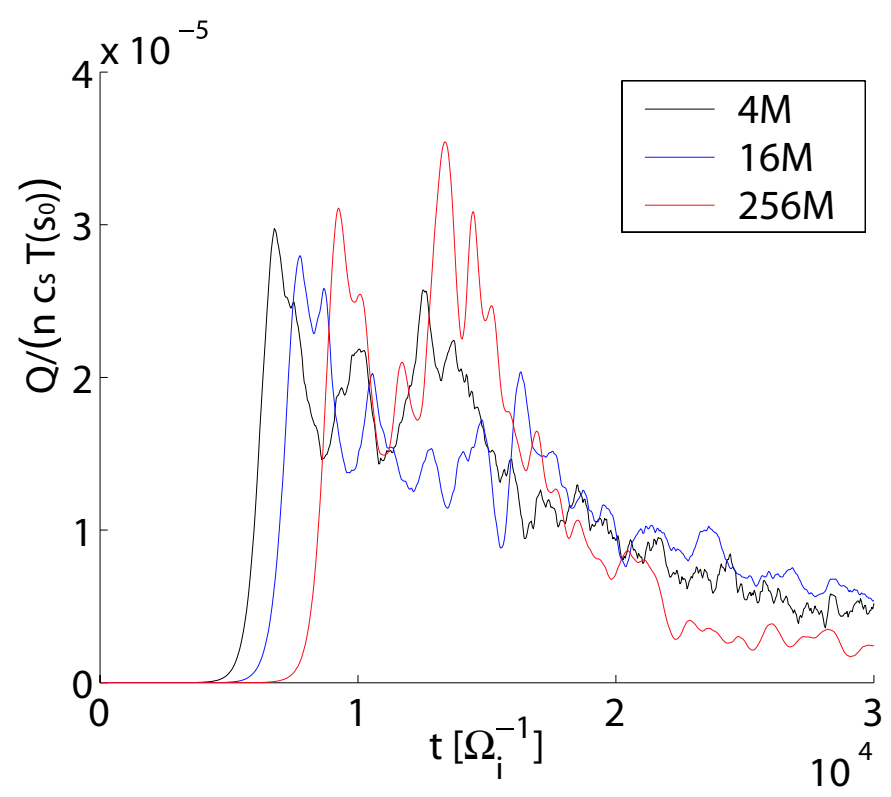

Figure 14. Time evolution of radial for different markers number with white noise initialization and a field-aligned filter.
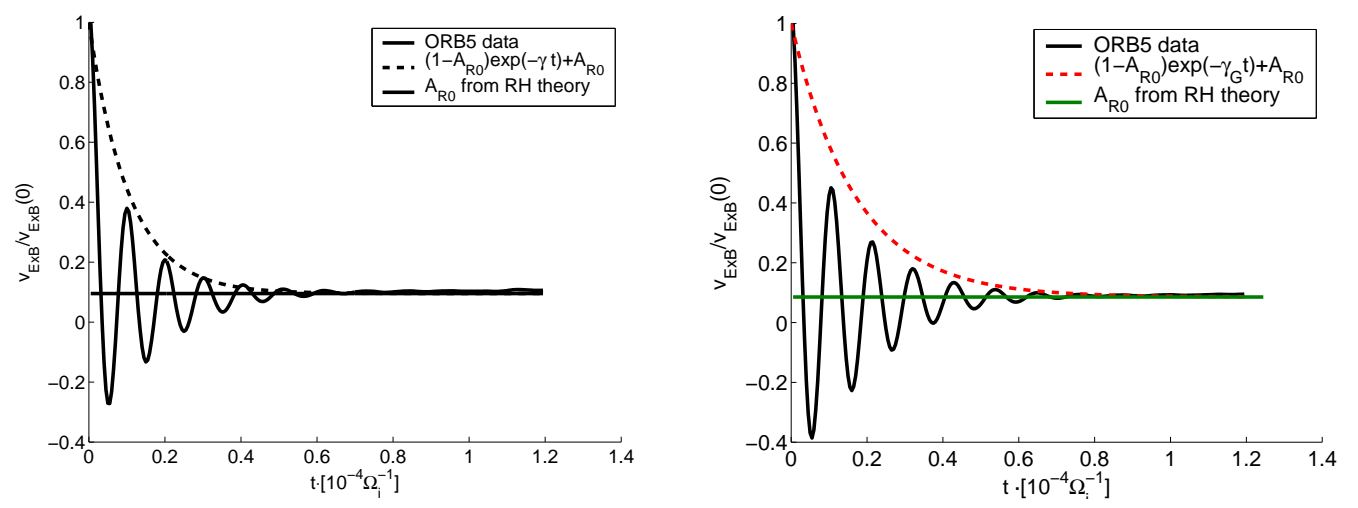

Figure 15. $\vec{E} \times \vec{B}$ velocity at $s_{0}=0.5$ (left) and $s_{0}=0.7$ (right) as a function of time. The solid line is the result of the ORB5 simulation in linear mode, with $\cos (\pi s)$ perturbation. The dotted line is the residual and the dashed line is the exponential decay from Rosenbluth-Hinton theory.

\begin{tabular}{|c|c|c|c|c|c|}
\hline pert & $s_{0}$ & $\omega_{G}$ (Eq. (54)) & code $\omega_{G}$ & $\gamma_{G}$ (Eq. (55)) & code $\gamma_{G}$ \\
\hline $\cos$ & 0.5 & 0.0059 & $0.0062 \pm 0.0002$ & -0.0009 & $-0.0009 \pm 0.0001$ \\
\hline $\cos$ & 0.7 & 0.0057 & $0.0058 \pm 0.0002$ & -0.0006 & $-0.0007 \pm 0.0001$ \\
\hline $\sin$ & 0.5 & 0.0059 & $0.0062 \pm 0.0002$ & -0.0009 & $-0.0008 \pm 0.0001$ \\
\hline $\sin$ & 0.7 & 0.0057 & $0.0058 \pm 0.0002$ & -0.0006 & $-0.0006 \pm 0.0001$ \\
\hline
\end{tabular}

Table 1

Comparison between analytical and numerical values for $\omega_{G}$ and $\gamma_{G}$. 

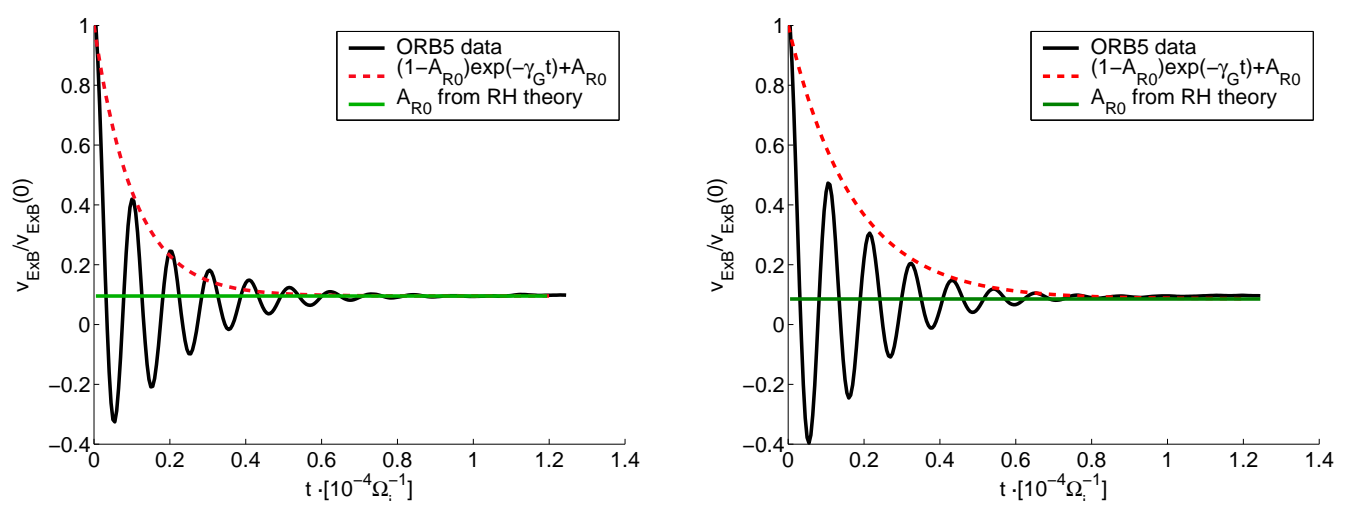

Figure 16. $\vec{E} \times \vec{B}$ velocity at $s_{0}=0.5$ (left) and $s_{0}=0.7$ (right) as a function of time. The solid line is the result of the ORB5 simulation in linear mode, with $\sin (\pi s)$ perturbation. The dotted line is the residual and the dashed line is the exponential decay from Rosenbluth-Hinton theory.

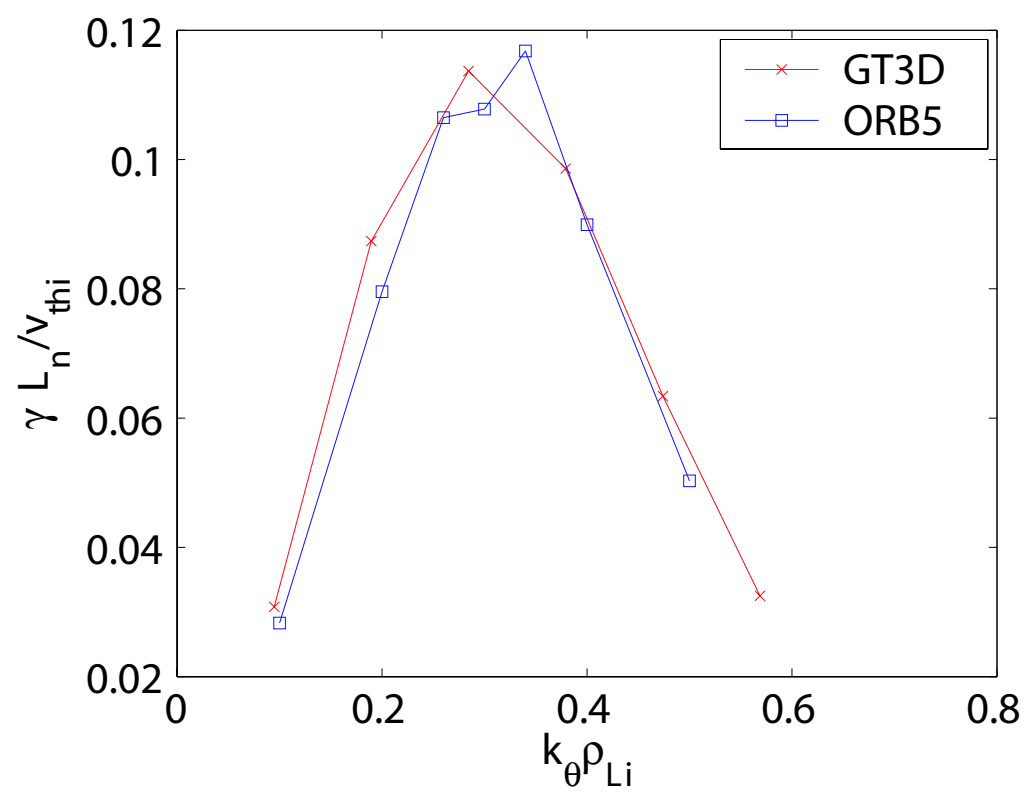

Figure 17. Linear growth rates for GT3D and ORB5. 


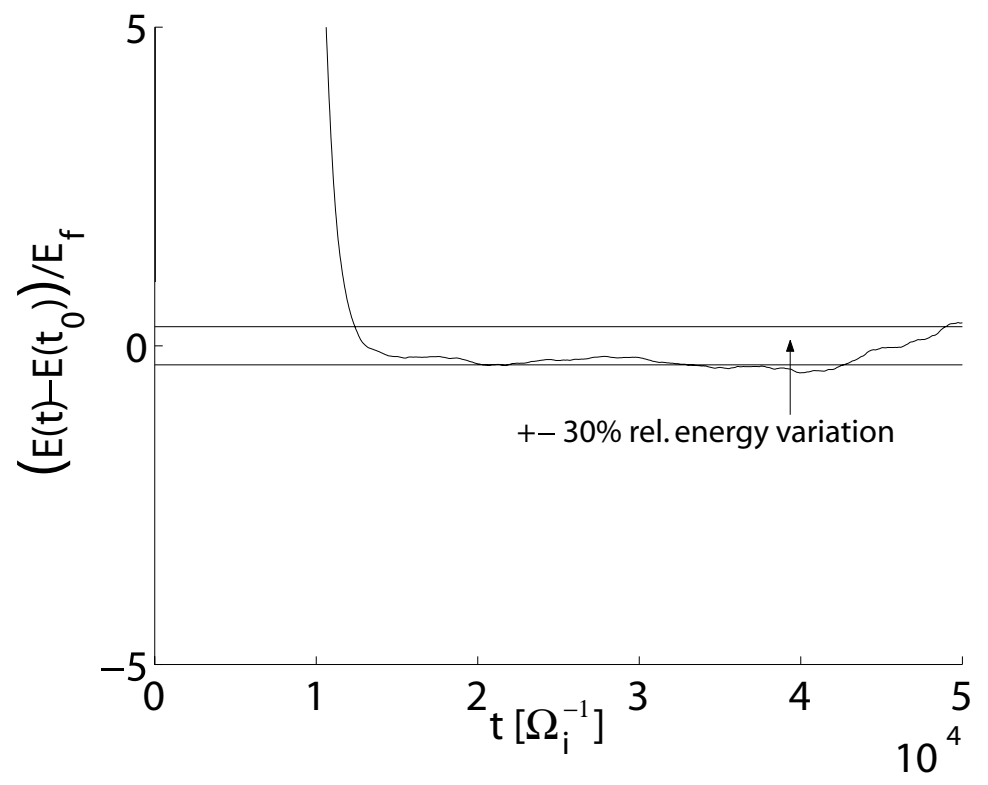

Figure 18. Relative energy conservation for a typical CYCLONE simulation.

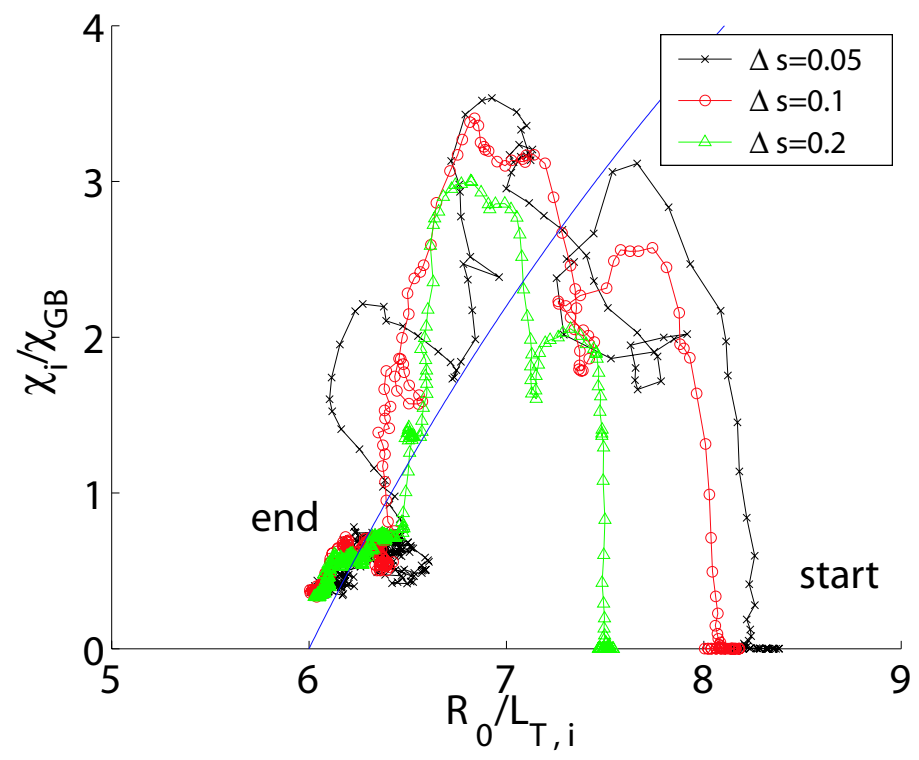

Figure 19. Evolution of $\chi_{i}$ vs $R_{0} / L_{T, i}$. Each point is averaged between $s_{0} \pm \Delta s$. A point is computed every $320 \Omega_{i}^{-1}$. The solid blue curve is the Dimits fit. 


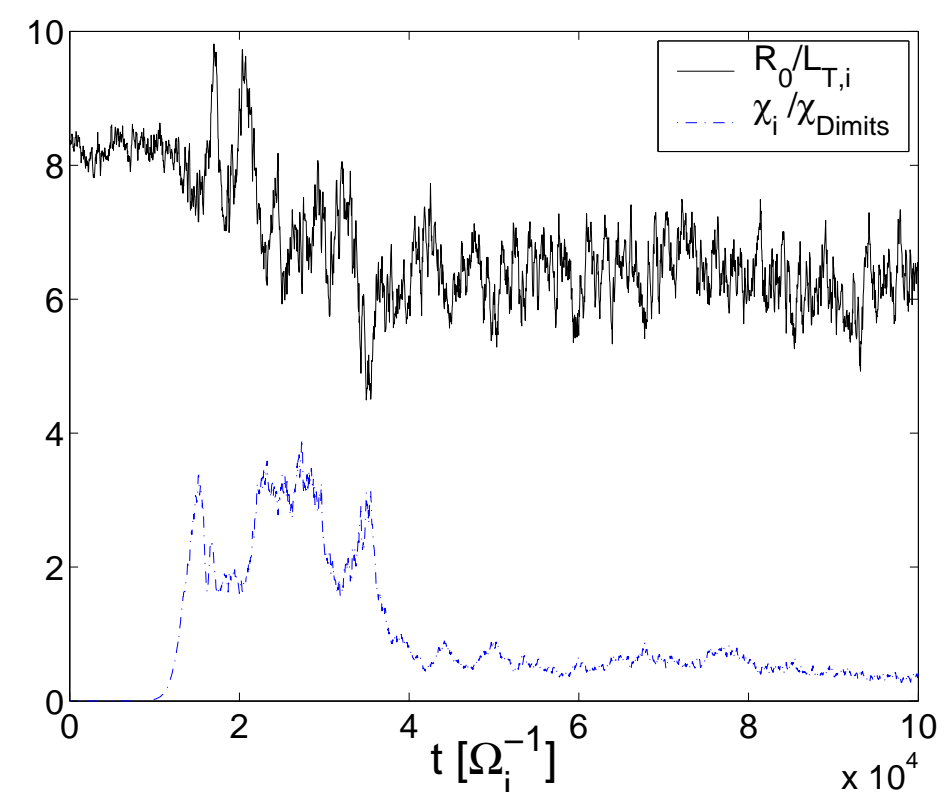

Figure 20. Time evolution of $R_{0} / L_{T, i}$ (blue) and $\chi_{i} / \chi_{\text {Dimits }}$ at $s=s_{0}$ 


\section{References}

[1] ITER Physics Expert Group on Confinement and Transport, iter Physics Expert Group on Confinement Modelling and Database, Iter Physics Basis Editors. Nucl. Fusion 39:2175 (1999)

[2] X. Garbet. Plasma. Phys. Control. Fusion 43:A251 (2001).

[3] W. Horton. Rev. Modern Phys. 71:735 (1999)

[4] P. H. Diamond, S.-I. Itoh, K. Itoh and T. S. Hahm. Plasma Phys. Control. Fusion 47:R35 (2005).

[5] L. Garcia and B. A. Carreras. Phys. Plasmas 12:092305 (2005).

[6] J. F. Drake, P. N. Guzdar and A. B. Hassam. Phys. Rev. Lett. 61:2205 (1988).

[7] F. Jenko, W. Dorland, M. Kotschenreuther and B. N. Rogers. Phys. Plasmas 7:1904 (2000).

[8] W. W. Lee. Phys. Fluids, 26:256 (1983).

[9] T. S. Hahm. Phys. Fluids. 31:2670 (1988).

[10] E. A. Frieman And L. Chen. Phys. Fluids 25: 502 (1982).

[11] A. J. Brizard. Phys. Plasmas 7:4816 (2000).

[12] R. G. Littlejohn J. Plasma Physics, 29:111 (1983).

[13] T. M. Tran, K. Appert, M. Fivaz, G. Jost, J. Vaclavik and L. Villard. Theory of fusion Plasmas, Int. Workshop, Editrice Compositori, Societá italiana di Fisica, Bologna, 1999, p. 45.

[14] A. M. Dimits, T. J. Williams, J. A. Byers and B. I. Cohen. Phys. Rev. Lett., $77: 71$ (1996).

[15] Z. Lin, T.S. Hahm, W. W. Lee, W. M. Tang and R. B. White. Science, 281, 1835 (1998).

[16] Y. Idomura, S. Tokuda And Y. Kishimoto. Nucl. Fusion 43:234 (2003).

[17] R. Sydora, V. Becyk and J. Bawson. Plasma Phys. Control. Fusion, 38:A281 (1996).

[18] S. E. Parker, C. Kim And Y. Chen. Phys. Plasmas 6:1709 (1999).

[19] G. Jost, T. M. Tran, W. A. Cooper, L. Villard and K. Appert. Phys. Plasmas 8, 3321 (2001).

[20] V. Kornilov, R. Kleiber, R. Hatzky, L. Villard and G. Jost. Phys. Plasmas 11:3196 (2004). 
[21] M. Kotschenreuther. Bull. Am. Phys. Soc., 33:2107 (1988).

[22] R. Hatzky, T. M. Tran, A. Könies, R. Kleiber and S. J. Allfrey. Phys. Plasmas, 9:898 (2002)

[23] P. Angelino, A. Bottino, R. Hatzky, S. Jolliet, T. M. Tran and L. Villard.Plasma Phys. Control. Fusion, 48:557 (2006).

[24] H. Lütjens, A. Bondeson and O. Sauter. Comp. Phys. Comm. 97:219 (1996).

[25] P. Angelino, A. Bottino, R. Hatzky, S. Jolliet, O. Sauter, T. M. Tran And L. Villard. Phys. Plasmas 13, 052304 (2006).

[26] S. J. Allfrey and R. Hatzky. Comp. Phys. Comm. 154:98 (2003.)

[27] C. K. Birdsall and A. B. Langdon. Adam Hilger, Bristol (1989).

[28] W. W. LeE. Journ. Comp. Phys., 72(1):243 (1987).

[29] K. J. Bowers. Journ. Comp. Phys., 173(2):393 (2001).

[30] M. Fivaz, S. Brunner, G. De Ridder, O. Sauter, T. M. Tran, J. Vaclavik, L. Villard and K. Appert. Comp. Phys. Commun., 111:27 (1998).

[31] K. HöLLIG. Finite Element Methods with B-Splines. Society for Industrial and Applied Mathematics (SIAM), Philadelphia (2003).

[32] S. Jolliet, P. Angelino, A. Bottino, Y. Idomura and L. Villard. Theory of fusion Plasmas, Int. Workshop, Editrice Compositori, Societá italiana di Fisica, Bologna, 2005, p. 345.

[33] A. Bottino, T. M. Tran, O. Sauter, J. Vaclavik and L. Villard. Theory of fusion Plasmas, Int. Workshop, Editrice Compositori, Societá italiana di Fisica, Bologna, 2001, p. 327.

[34] S. Jolliet, A. Bottino, P. Angelino, T. M. Tran, B. F. Mcmillan, R. Hatzky, A. G. Peeters, E. Poli, O. Sauter and L. Villard. Proceedings of Theory of fusion Plasmas, 2006, to be published.

[35] C. C. Kim and S. E. Parker. Journ. Comp. Phys., 161(2):589 (2000).

[36] R. Hatzky. Parallel Computing 32:325 (2006).

[37] S. Brunner. PhD Thesis No.1701, Ecole Polytechnique Fédérale de Lausanne, (1997).

[38] A. Bottino, P. Angelino, R. Hatzky, S. Jolliet, A. G. Peeters, E. Poli, O. Sauter, T. M. Tran and L. Villard Proc. $33^{\text {rd }}$ EPS Conf. on Plasma Physics and Controlled Fusion (Roma, Italy, June 2006), to be published.

[39] W. M. Nevins, G.W. Hammett, A.M. Dimits, W. Dorland and D.E. Shumaker. Phys. Plasmas 122305 (2005). 
[40] M. N. Rosenbluth And F. L. Hinton. Phys. Rev. Lett., 80:724 (1998).

[41] N. Winsor, J. L. Johnson and J. M. Dawson. Phys. Fluids, 11:2448 (1968).

[42] C. M. Greenfield, J. C. DeBoo, T. H. Osborne, F. W. Perkins, M. N. Rosenbluth And D. Boucher. Nucl. Fusion, 37:1215 (1997).

[43] A. M. Dimits, G. Bateman, M. A. Beer, B. I. COhen, W. Dorland, G. W. Hammet, C. Kim, J. E. Kinsey, M. Kotschenreuter, A. H. Kritz, L. L. Lao, J. Mandrekas, W. M. Nevins, S. E. Parker, A. J. Redd, D. E. Shumaker, R. Sydora And J. Weiland.Phys. Plasmas 7:969 (2000).

[44] S. P. Hirshman, D. K. Lee, F. M. Levinton, S. H. Batha, M. Okabayashi And R. M. Wieland. Phys. Plasmas 1, 2277 (1994).

[45] R. E. Waltz, J. C. DeBoo and M. N. Rosenbluth. Phys. Rev. Let. 65: 2390 (1990).

[46] B. D. Scott. Phys. Plasmas 12: 102307 (2005).

[47] J. Candy, R. E. Waltz and W. Dorland. Phys. Plasmas 11:L25 (2004).

[48] Z. Lin, S. Ethier, T. S. Hahm and W. M. Tang. Phys. Rev. Lett. 88, 195004 (2002). 